\title{
An advanced joint time-frequency analysis procedure to study cavitation-induced noise by using standard series propeller data
}

\author{
Batuhan Aktas *, Mehmet Atlar, Patrick Fitzsimmons, Weichao Shi \\ Naval Architecture, Ocean \& Marine Engineering, University of Strathclyde
}

Glasgow, UK

Corresponding Author:

*Batuhan Aktas, batuhan.aktas@strath.ac.uk

Naval Architecture, Ocean \& Marine Engineering,

Henry Dyer Building, 100 Montrose Street

University of Strathclyde, G4 0LZ

Glasgow, UK

Tel: $\quad 01415745342$

\begin{abstract}
:
Underwater noise has attracted significant amount of interest in the last decade because of its potential impact on marine fauna. Commercial shipping is one of the major contributors. Amongst various sources on-board, cavitation dominates the overall radiated noise levels beyond its inception. Besides its significance, the mechanisms driving cavitation related noise and frequency regions that certain cavitation dynamics contribute into are not studied sufficiently. To address this gap in the literature, a study has been conducted to provide means of a plausible propeller cavitation noise prediction method and to provide enhanced insight to the noise creating mechanisms of cavitation by applying appropriate signal processing methods.
\end{abstract}


Within this framework this study presents an advanced joint time-frequency analysis procedure to study cavitation-induced noise by using standard series propeller data. Systematic cavitation tunnel test are conducted with 6 members of the Meridian Standard propeller series that are carefully chosen to study the influence of major propeller design parameters such as Blade Area Ratio (BAR), Pitch to Diameter (P/D) ratio and blade number. Moreover, these propellers are tested behind systematically varied wake inflows reproduced by wake screens in addition to the open water conditions. Synchronized pressure pulse and noise measurements in combination with high-speed cavitation observations are then utilized to develop an advanced cavitation dynamics analysis tool to provide better insight into cavitation-induced noise. The analysis of the measurements has shown the significance of the cavitation on radiated noise levels and the impact of different cavitation dynamics in certain frequency regions.

Keywords: Underwater Radiated Noise; Propeller Cavitation Noise; Experimental Hydrodynamics; Joint Time-Frequency Analysis

\section{Nomenclature:}

$\begin{array}{ccc}A_{E} & \text { Expanded blade area }\left[\mathrm{m}^{2}\right] & R \\ A_{o} & \text { Propeller disc area }\left[\mathrm{m}^{2}\right] & R_{A} \\ \text { BAR } & \text { Blade Area Ratio } & r_{\text {ref }} \\ D & \text { Diameter }[\mathrm{m}] & r \\ f & \text { Frequency }[\mathrm{Hz}] & \rho \\ g & \text { Gravitational acceleration }\left[\mathrm{m} / \mathrm{s}^{2}\right] & S P L \\ h_{S} & \text { Shaft immersion }[\mathrm{m}] & T \\ J & \text { Advance coefficient } & U R N \\ K_{P} & \text { Pressure coefficient } & V \\ K_{Q} & \text { Torque coefficient } & V_{A} \\ K_{T} & \text { Thrust coefficient } & W_{\text {Max }} \\ N & \text { Rotational speed }[\mathrm{rpm}] & W_{\Delta} \\ n & \text { Propeller revolution speed }[\mathrm{rps}] & \bar{W} \\ P & \text { Pressure }\left[\mathrm{N} / \mathrm{m}^{2}\right] & W_{\text {Min }} \\ P / D & \text { Pitch ratio at } 0.7 R & Z \\ P_{\text {atm }} & \text { Atmospheric pressure }\left[\mathrm{N} / \mathrm{m}^{2}\right] & \eta_{o} \\ P_{v} & \text { Vapour Pressure }\left[\mathrm{N} / \mathrm{m}^{2}\right] & \sigma_{v r} \\ Q & \text { Torque }[\mathrm{Nm}] & \end{array}$

Radius $[\mathrm{m}]$

Distance between the acoustic center of the noise source and hydrophone $[\mathrm{m}]$ Reference distance $(1 \mathrm{~m})[\mathrm{m}]$

Local propeller radii [m]

Fluid density $\left[\mathrm{kg} / \mathrm{m}^{3}\right]$

Sound pressure level [dB]

Thrust [N]

Underwater Radiated Noise

Speed $[\mathrm{m} / \mathrm{s}]$

Advance speed $[\mathrm{m} / \mathrm{s}]$

Maximum wake fraction

BSRA Wake Criteria (wake non-unormity

parameter)

Mean wake

Minimum wake fraction

Propeller blade number

Propeller efficiency

Resultant cavitation number 



\section{Introduction}

Commercial shipping has revolutionized today's world by providing the most efficient means of transportation (Stopford, 1997). The volume of commercial shipping has experienced an increasing trend due to increasing ship size, service speed and number of ships in operation. With the rising trend in commercial shipping, there has been a significant rise in various emissions from ocean-going vessels. One of the most adverse by-products of the commercial shipping has been underwater noise emission. The extraordinary expansion of the world fleet has resulted in increased levels of the ambient noise in the world's seas, especially in the low frequency domain (Frisk, 2012). Unfortunately, this domain overlaps with that used by marine mammals for various fundamental living activities. Thus, exposing them to such an abrupt change in ambient noise levels may disorient them or disrupt their communication signals, leading to behavioural changes of these mammals or local extinction (Richardson et al., 2013; White and Pace, 2010).

Amongst numerous ship sources, propeller cavitation dominates the overall radiated noise spectrum above the inception threshold. Whilst it is not possible to avoid cavitation for commercial ships at current service speeds, without trading off efficiency, various full-scale underwater radiated noise (URN) measurements in the literature have shown that improvements can be achieved. Among the same types of vessel, full-scale measurements have shown up to $20 \mathrm{~dB}$ difference in the measured noise levels (McKenna et al., 2013; MCR, 2011; Wales and Heitmeyer, 2002).

Propeller cavitation noise prediction is best achieved through accurate and reliable cavitation tunnel experiments at an early design stage. Even if invaluable cavitation data and associated other performance data (e.g. fluctuating hull pressures, propeller performance characteristics) can be obtained from these experiments, which can be extrapolated to full-scale using suitable scaling procedures, there is still a need to interpret some physical complexities associated with cavitation dynamics in model scale. The effect of cavitation, and particularly the dynamics of cavitation, on URN is rather complex to understand and the mechanisms driving cavitationrelated noise and frequency regions that certain cavitation dynamics contribute into are not studied sufficiently. Within this framework, the aim of this paper is to enhance understanding of the propeller cavitation noise by conducting detailed systematic cavitation tunnel tests by 
developing an advanced cavitation dynamics analysis tool using pressure pulse and noise measurements that are synchronized with high-speed cavitation observations.

Experimental noise studies with the propellers are of great importance as they represent the state of the art and lead towards the knowledge gaps within the literature. Until the recent doctoral study conducted by the Principal Author of this paper (Aktas, 2017) there has been no study published that has used standard propeller series for noise investigations with systematical wake variation. Some studies have compared the effect of some major propeller designs and operational parameters on the radiated noise levels as well as the effect wake inflow. In his early study, Holden, (Holden, 1981), carried out an experimental campaign with six propellers out of four that have been tested in the same wake field and two in different inflow conditions. The study set out to investigate the effect of BAR, radial camber and pitch distribution and skew with the four propellers and to look into the effect of wake inflow with an additional two propellers. This work reported that higher BAR, tip offloading and skew reduced the emitted noise levels originating from the propeller cavitation. While the tests have led to some significant conclusions regarding the major propeller design parameters, it failed to take into account wake inflow variations. Moreover, it is not known if the propellers tested were members of a standard propeller series. Whilst detailed pitch and camber distributions are provided for all propellers, there is no mention to their blade section shapes.

Sharma et al., (Sharma et al., 1990) conducted extensive experiments with three basic propellers models of different design to investigate the effect of advance coefficient, cavitation number and propeller geometry on radiated noise levels in uniform flow conditions. Furthermore, one of the propellers was tested behind a typical single-screw vessel wake which led to the conclusion that wake is one of the most important parameters for noise generation. Although the study provides valuable information and is one of the most important studies conducted on this subject, the work was limited to testing three independent propellers with no systematic variation of parameters. Thus, no relation can be drawn from this study with regards to the influence of major propeller parameters on the radiated noise levels of ships.

An influential study by Konno et al., (Konno et al., 2002) studied sheet and tip vortex dynamics phenomena. The research considered the modern propeller design tendency to use highly skewed propellers for lowering pressure fluctuations. However, the skew distribution created a strong cavitating tip vortex which emitted high levels of acoustic pressure when deforming within the wake peak. The experimental strategy applied in the study of Konno et al. is centred 
on the effects of various parameters on tip vortex cavitation dynamics (the so-called bursting phenomenon). One of the most important aspect of this study was the inclusion of four wake variations. The severity of each wake was determined by means of the wake fraction gradients (see Figure 1a). The developed wakes were tested with two propellers having different pitch distributions as shown by Figure 1b. The experiments were conducted at different cavitation numbers and thrust coefficients in order to assess the influences of such parameters.

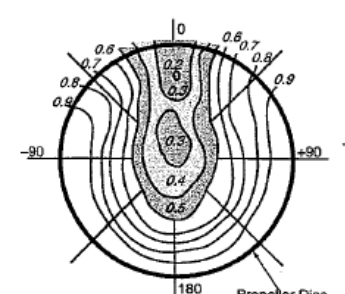

Wake W1

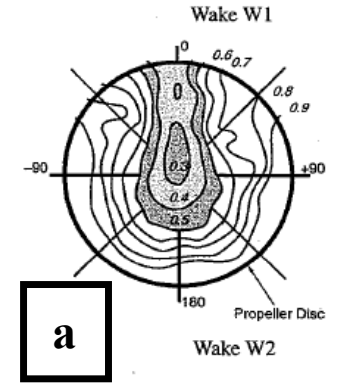

Figure 1 Wake non-uniformities (a)

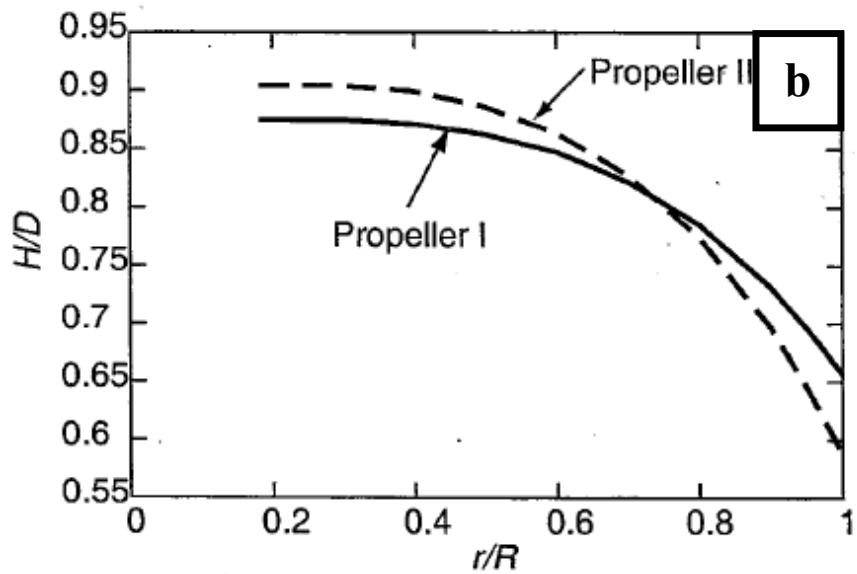

The study by Konno et al., (Konno et al., 2002) draws some remarkable conclusions on the effects of such variants on the radiated noise levels. First of all, the wake inflow has a high influential on the radiated noise levels and the results of this study confirm that the cavitation noise increases with the increasing wake severity. Moreover, the study also indicates that the measured levels increase over the whole frequency range, as expected, with the decreasing cavitation number. Whilst certain trends are observed for the thrust coefficient and propeller pitch distribution they are more complex in nature due to the highly irregular cavitation interaction and dynamics. Although this work provides some vital evidence of how propeller cavitation noise changes with respect to propeller pitch distribution and operational parameters in conjunction with the flow non-uniformity alteration, it is limited by the absence of tests regarding the major propeller design parameters and by the small number of test conditions conducted.

Advanced analysis techniques have been utilized for propeller cavitation and noise studies. Pereira et al., (2004) performed harmonic analysis using high-speed visualizations, pressure 
and noise measurements around a cavitating propeller. This study was conducted in a nonuniform flow field to identify different pressure and noise sources and their respective contribution to the induced fluctuation. In the same vein, (Pennings et al., 2016), simultaneous high-speed video shadowgraphy and noise measurements are used to analyse the noise radiated by tip vortices. An example of time-frequency analysis, through the wavelet transform, used to analyze the pressure fluctuations produced by tip vortices is reported by Bosschers, (2009).

In the light of the reviewed literature, it is evident that a study demonstrating experimental quantitative pressure data analysis combined with qualitative cavitation observation is not publicly available for a standard series of propellers with a systematically varied non-uniform inflow. The studies reviewed here point to a knowledge gap in terms of analysis methodology of the hydro-acoustic propeller cavitation tests. The research to date has demonstrated a number of experimental studies on cavitation noise, however, there is no single study that demonstrates an advanced cavitation noise analysis methodology to enable systematic investigation of the propeller cavitation noise. To address this gap, Aktas (Aktas, 2016) has conducted a comprehensive experimental study by using standard series model propellers to develop a procedure in predicting URN from commercial ship propellers as well as an advanced joint time-frequency analysis (JTFA) procedure for cavitation dynamics by making use of the systematic data produced in this experimental campaign. The JTFA procedure is to understand the effect of cavitation dynamics on the cavitation-induced URN by using synchronised pressure pulses, high-speed cavitation images and underwater noise signals. The post processing tool clearly indicated that cavitation is the dominating noise source following its inception. The wake non-uniformity is the vital parameter to consider for the standard series propeller noise tests since the cavitation dynamics experienced due to the flow irregularity is one of the main sources of the emanated noise levels. Moreover, the cavitation type experienced by any propeller is highly influential over the emanated noise levels as well as the frequency region for which certain type of cavitation contributes into.

This paper presents the details of this procedure and its application on the systematic experimental data produced in four sections. Following this introduction, section 2 explains the adopted experimental approach. Section 3 then presents the results of the noise measurements and demonstrates an advanced analysis of cavitation-induced noise data using the JTFA 
technique. Finally, major conclusions drawn from the study about the procedure are given in section 4.

\section{Adopted experimental approach}

The experimental approach adopted necessitated the use of several experimental artefacts and as the main testing facility, a cavitation tunnel. The Emerson Cavitation Tunnel (ECT) is a closed circuit depressurized tunnel with a measuring section of $3.1 \mathrm{~m}$ x $1.21 \mathrm{~m}$ x $0.8 \mathrm{~m}(\mathrm{LxBxH})$. More detailed information about the tunnel in detail after the recent upgrade is given by Atlar (Atlar, 2011). Details of the selected standard series are presented in section 2.1 while the description of the representative wake inflows and their constructions for the experiments are given in section 2.2. Finally in section 2.3, detailed information about the experimental setup for the URN tests is given.

\subsection{Selection of standard propeller series}

Newcastle University with its ECT facility has been the birthplace of a number of famous and widely used systematic propeller series (e.g. KCA or Gawn-Burrill, KCD, Meridian, etc.) for which comprehensive open water performance charts have been published (Emerson and Sinclair, 1978; Gawn and Burrill, 1957). Some of these data were used in the development of Burrill's cavitation criterion (Burrill and Emerson, 1962) which relates sheet cavity extent to thrust, blade area and cavitation number. Such historical data have formed the background to the main objective of the present study, namely, to generate a representative systematic body of underwater noise data for a subset of the standard "Meridian propeller series".

Propeller design with the absence of computational power initially relied mainly on experience and model tests. Eventually, in order to aid propeller designers, standard propeller series were developed and tested to give guidelines and a design methodology (Kuiper, 2010). These series have enabled the propeller designer to quickly size the propeller using charts whilst ensuring with sufficient thrust to achieve the contract speed and power. Table 1 (Carlton, 2012) shows some of the well-known standard series of propellers. The Emerson Cavitation Tunnel has been involved in the development of the KCA series with the support and funding of the British Admiralty and both the KCD and Meridian series with Stone Marine Manganese (Emerson and Sinclair, 1978; Gawn and Burrill, 1957). 
Table 1 Fixed-pitch, non-ducted propeller series (Reproduced from(Carlton, 2012))

\begin{tabular}{|c|c|c|c|c|c|c|c|c|}
\hline \multirow[t]{2}{*}{ Series } & \multirow{2}{*}{$\begin{array}{l}\text { Number of } \\
\text { propellers } \\
\text { in series }\end{array}$} & \multicolumn{3}{|c|}{ Range of parameters } & \multirow[t]{2}{*}{$D(\mathrm{~mm})$} & \multirow[t]{2}{*}{$r_{h} / R$} & \multirow{2}{*}{$\begin{array}{l}\text { Cavitation } \\
\text { data } \\
\text { available }\end{array}$} & \multirow[t]{2}{*}{ Notes } \\
\hline & & $\mathrm{z}$ & $A_{\mathrm{E}} / A_{\mathrm{O}}$ & $P / D$ & & & & \\
\hline $\begin{array}{l}\text { Wageningen } \\
\text { B-series }\end{array}$ & $\simeq 120$ & $2-7$ & $0.3-1.05$ & $0.6-1.4$ & 250 & 0.169 & No & $\begin{array}{l}\text { Four-bladed } \\
\text { propeller has } \\
\text { non-constant } \\
\text { pitch dist }\end{array}$ \\
\hline Au-scries & 34 & $4-7$ & $0.4-0.758$ & $0.5-1.2$ & 250 & 0.180 & No & \\
\hline Gawn-series & 37 & 3 & $0.2-1.1$ & $0.4-2.0$ & 508 & 0.200 & No & \\
\hline KCA-series & $\simeq 30$ & 3 & $0.5-1.25$ & $0.6-2.0$ & 406 & 0.200 & Yes & \\
\hline Ma-series & 32 & 3 and 5 & $0.75-1.20$ & $1.0-1.45$ & 250 & 0.190 & Yes & \\
\hline $\begin{array}{l}\text { Newton-Rader } \\
\text { series }\end{array}$ & 12 & 3 & $0.5-1.0$ & $1.05-2.08$ & 254 & 0.167 & Yes & \\
\hline KCD-series & 24 & $\begin{array}{l}3-6 \\
\text { (mainly 4) }\end{array}$ & $\begin{array}{l}0.587 \text { Principal } \\
0.44-0.8\end{array}$ & $0.6-1.6$ & 406 & 0.200 & Yes & $\begin{array}{l}\text { Propellers not } \\
\text { geosyms }\end{array}$ \\
\hline Meridian serics & 60 & $4-6$ & $0.45-1.05$ & $0.4-1.2$ & 305 & 0.185 & Yes & $\begin{array}{l}\text { Propellers not } \\
\text { geosyms }\end{array}$ \\
\hline
\end{tabular}

Amongst the various standard propeller series that are readily available in the inventory of the ECT, the one with the best coverage in terms of the major propeller design parameters for acoustic testing is considered to be the Meridian Standard Propeller series (Emerson and Sinclair, 1978). This family of propellers also has existing propeller performance data that enables cross checking of measurement reliability.

The Meridian propeller series is a unique standard series based solely on practical propeller designs for standardised variations in pitch to diameter ratio, BAR and number of blades (Z). The series is unique in that actual propeller designs were chosen as the parent propellers for each blade number group and blade area group. These were largely obtained from the extensive database of Stone Marine Propulsion (SMP).

\subsection{Development of systematic variation of wake inflow}

The main noise-producing mechanism during the operation of a marine propeller has been identified by several authors as the variation of cavitation volume (sheet and vortex) in a nonuniform wake field produced by the hull-form and appendages ahead of the propeller (Breslin and Andersen, 1996; Bretschneider et al., 2014; Pereira et al., 2004; Ross, 1976). This important effect has been included in this study through a systematic variation of wake inflow fields, constructed prior to conducting the cavitation tests. The synthesised wake characteristics were influenced by the British Ship Research Association (BSRA) wake criteria (Odabasi and Fitzsimmons, 1978) and important parameters of this criteria were identified in the BMT empirical noise prediction study (Angelopoulos et al., 1988). 
The significance of the hull wake and its distribution on propeller performance, especially for cavitation and inboard noise, has been noted in many research studies since the 1970's and is routinely taken into account in the design and analysis process, e.g (Oossanen, 1971; Young and Kinnas, 2001). Although all three components of the hull wake flow are important on the performance analysis of a propeller, the axial wake is the most important component dominating the propeller's loading characteristics. Consequently, it was decided that the desirable wake configurations could be simulated by using two-dimensional wake screens in combination with different grades of "chicken-wire" meshes, strategically patched onto a base frame which is in line with the established practice within the ECT.

In order to select suitable wake configurations, a literature survey was conducted. These included seminal studies such as Konno (Konno et al., 2002) in which cavity collapse events were observed to increase amplitudes and complexities of the pressure pulse time-series for the propeller operating behind a peaked, non-uniform inflow. The study further highlighted the effect of altering the gradient of the wake distribution (Konno et al., 2002). The wakes with steeper velocity changes were shown to produce higher tonal amplitudes of pressure fluctuations as well as high-frequency contributions from increased dynamic cavity collapses, both on and away from the blade surface. A characteristic pressure pulse feature was a double peak configuration in the time domain signals. Inspiration from Konno's study led to the utilization of BSRA wake criteria suggested by (Angelopoulos et al., 1988; Odabasi and Fitzsimmons, 1978) to quantify and develop a family of three representative wake grids, to complement the parametric variations in the standard propeller series geometries. According to Odabasi \& Fitzsimmons (Odabasi and Fitzsimmons, 1978), the severity of the wake, which can be quantified by the wake non-uniformity parameter given by Equation 1, is an important characteristic in generating propeller-excited vibrations (of the hull) and underwater radiated noise.

$$
\mathrm{W}_{\Delta, 0.7 r / R}=\left\{\frac{W_{\text {Max }}-W_{\text {Min }}}{1-\bar{W}}\right\} \quad \text { Equation } 1
$$


where, $W_{\text {Max }}$ and $W_{\min }$ are the maximum and minimum wake fraction and $\bar{W}$ is the circumferential average wake fraction calculated by Equation 2. The above is defined at $r / R=$ 0.7 in this study. Whilst the BSRA wake criteria normally is defined at $r / R=1.0$, the wake parameters at $r / R=0.7$ is preferred in the study. This was due to two reasons. First of all, the developed wake grids presented higher gradients at this radius and hence better representation of the implemented wake variation. Also, as demonstrated through the sample cavitation observations presented within this study, large extent sheet cavitation occurrence is known to concentrate generally at $\mathrm{r} / \mathrm{R}=0.7$.

$$
\bar{W}=\frac{\int_{r_{h u b}}^{R} r \int_{0}^{2 \pi} w_{T} d \emptyset d r}{\pi\left(R^{2}-r^{2}\right)}
$$

Equation 2

where, $r$ is the representative radius of the propeller that is integrated over the propeller radius, $w_{T}$ is the wake fraction that is integrated over the $360^{\circ}, \emptyset$ is the angle and $\mathrm{R}$ is the radius of the propeller.

In order to have a better definition and control of the systematic wake variation, further wake parameters such as the mean wake, half-wake width and wake depth $(\Delta \mathrm{W})$ have been included in the wake analysis as shown in Figure 2 for $r / R=0.7$. The highest value of the wake depth and lowest value of the half-wake width would present the most non-uniform wake distribution. This resulted in the most dramatic change of the inflow velocity in the wake shadow region and consequently would induce the formation of unsteady cavitation in this region by the collapse and rebound of cavity volumes at exit from the wake peak region.

Based on the above considerations, three related wake configurations were generated numerically (theoretically); these are designated as ECT1, ECT2 and ECT3, for varying wake non-uniformity parameters. The target variations are achieved through variation of the half wake width and wake depth parameters, ranging from severe to mild. Thus the wake with the narrowest wake width and highest wake depth presents the most severe wake (ECT1) and the wake that has the lowest wake depth and largest half wake width represents the mildest wake (ECT3) while ECT2 is lying in between. Table 2 shows the main parameters of the three wake configurations, while Figure 3 compares the target wake variations (dotted lines) with the experimentally simulated wake distributions (solid lines) at one fractional radius $(r / R=0.7)$. Cavitation dynamics outside the $0.7 \mathrm{r} / \mathrm{R}$ are considered to dominate noise generation. Outside 
the $0.7 \mathrm{r} / \mathrm{R}$ (i.e. $0.9 \mathrm{r} / \mathrm{R}$ and $1.1 \mathrm{r} / \mathrm{R}$ ) similar wake trends are also observed. $0.9 \mathrm{r} / \mathrm{R}$ and $1.1 \mathrm{r} / \mathrm{R}$ have strong influence on the stability of the tip vortex cavitation.

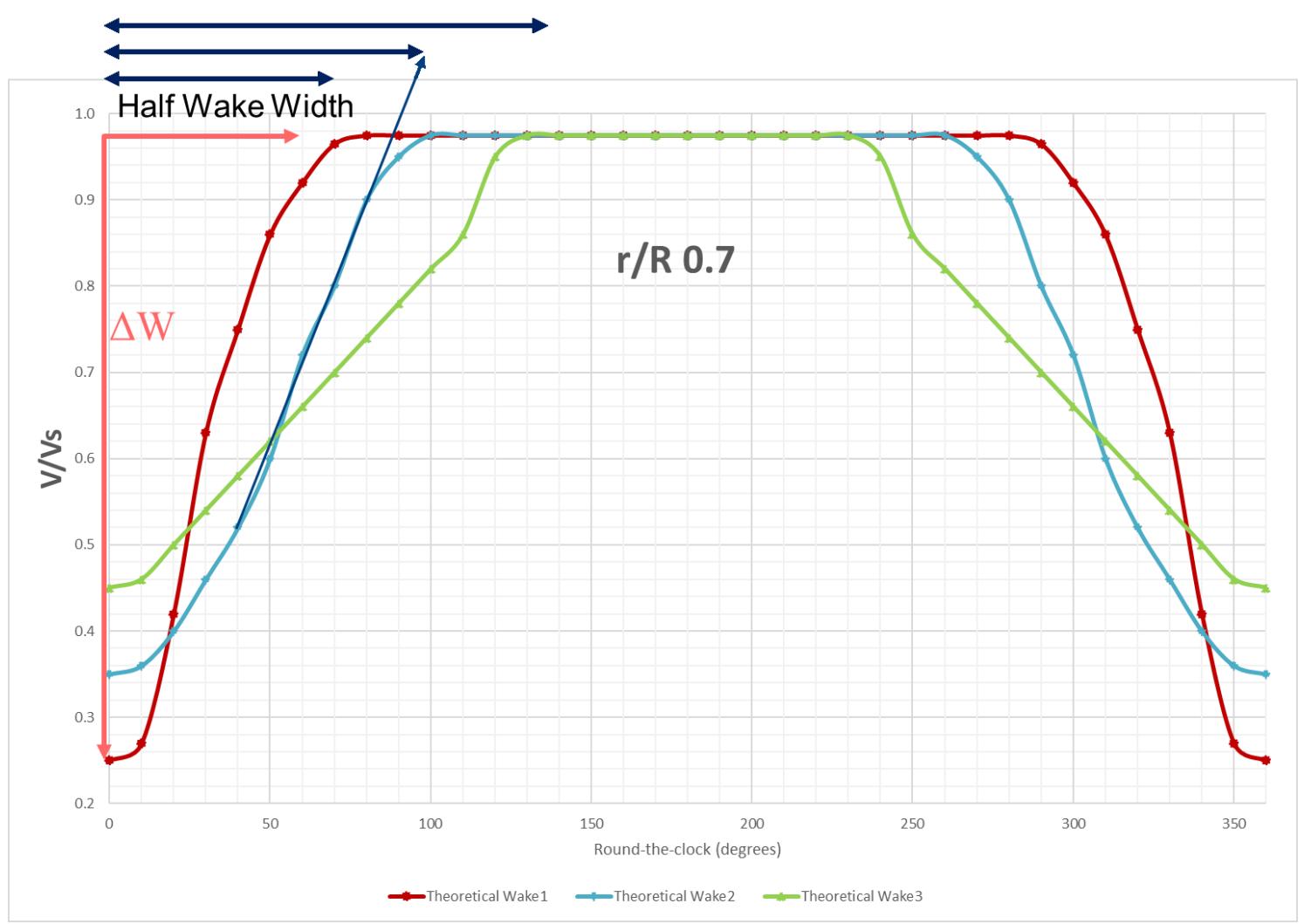

Figure 2 Representation of wake depth $(\Delta \mathrm{W})$ and half wake width for three representative wake distributions at $\mathbf{r} / \mathbf{R}=\mathbf{0 . 7}$. 


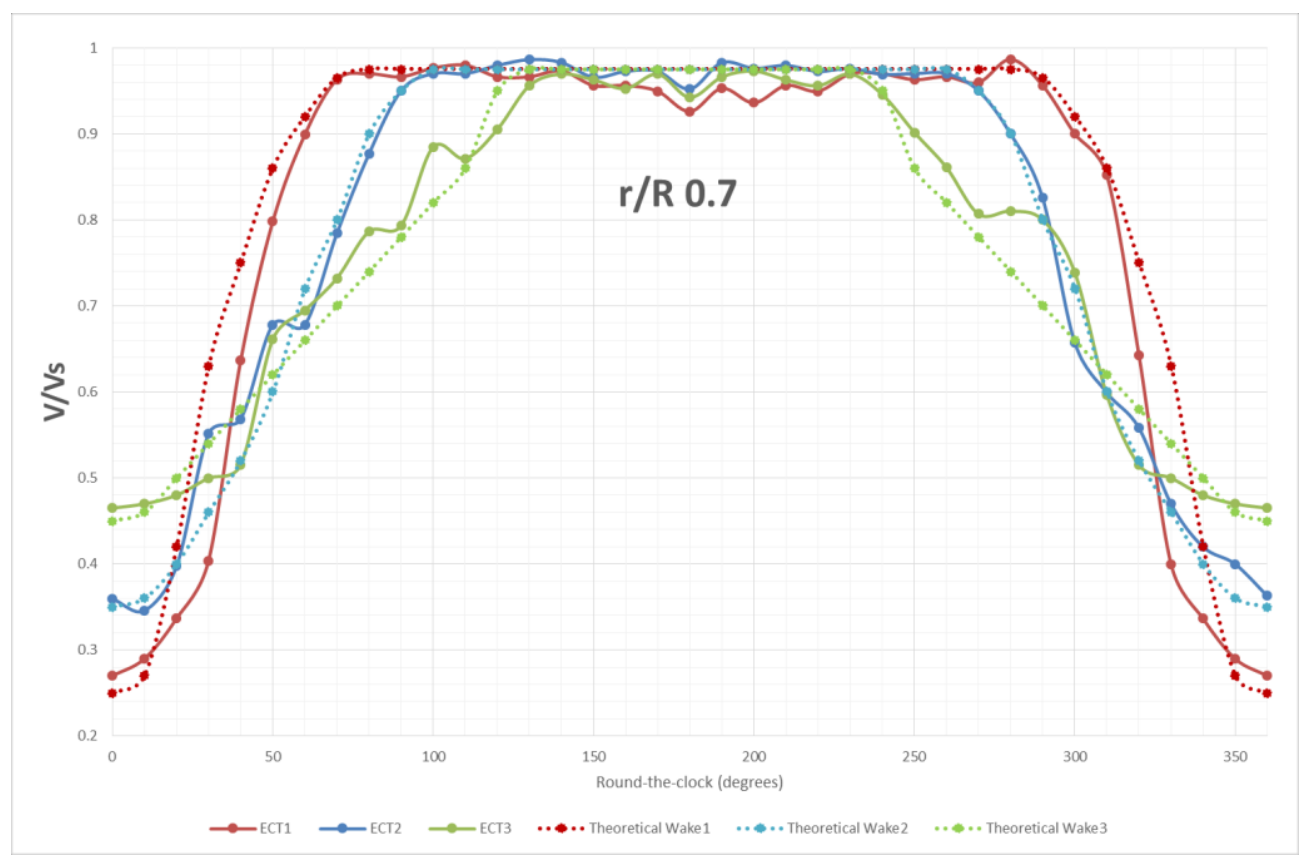

Figure 3 Comparison of target (theoretical) and simulated wakes (ECT) at $\mathbf{r} / \mathbf{R}=0.7$

Table 2 Significant wake parameters for wake distributions selected

\begin{tabular}{|l|c|c|c|}
\hline \multicolumn{3}{|c|}{ Wake Parameters } \\
\hline Wake screen type & ECT1 & ECT2 & ECT3 \\
\hline Wake depth & 0.71 & 0.64 & 0.50 \\
\hline Half Wake Width (degrees) & 60 & 95 & 132 \\
\hline Mean Wake (Propeller disc) & 0.16 & 0.20 & 0.195 \\
\hline Wake non-uniformity parameter & 0.86 & 0.81 & 0.63 \\
\hline
\end{tabular}

The flow measurements for the systematic variations of axial wakes using wake screens is conducted using an Laser Doppler Anemometry (LDA) unit set up, the results of which are run through BSA Flow Software. A measuring grid is defined at 1.5 propeller diameters downstream of the wake screen, as it is the propeller location, and included points on the nondimensional radius $(\mathrm{r} / \mathrm{R})$ values of $0.3,0.5,0.7,0.9,1.1$ for every 10 degrees making up a total of 180 point measurements as shown in Figure 4. 


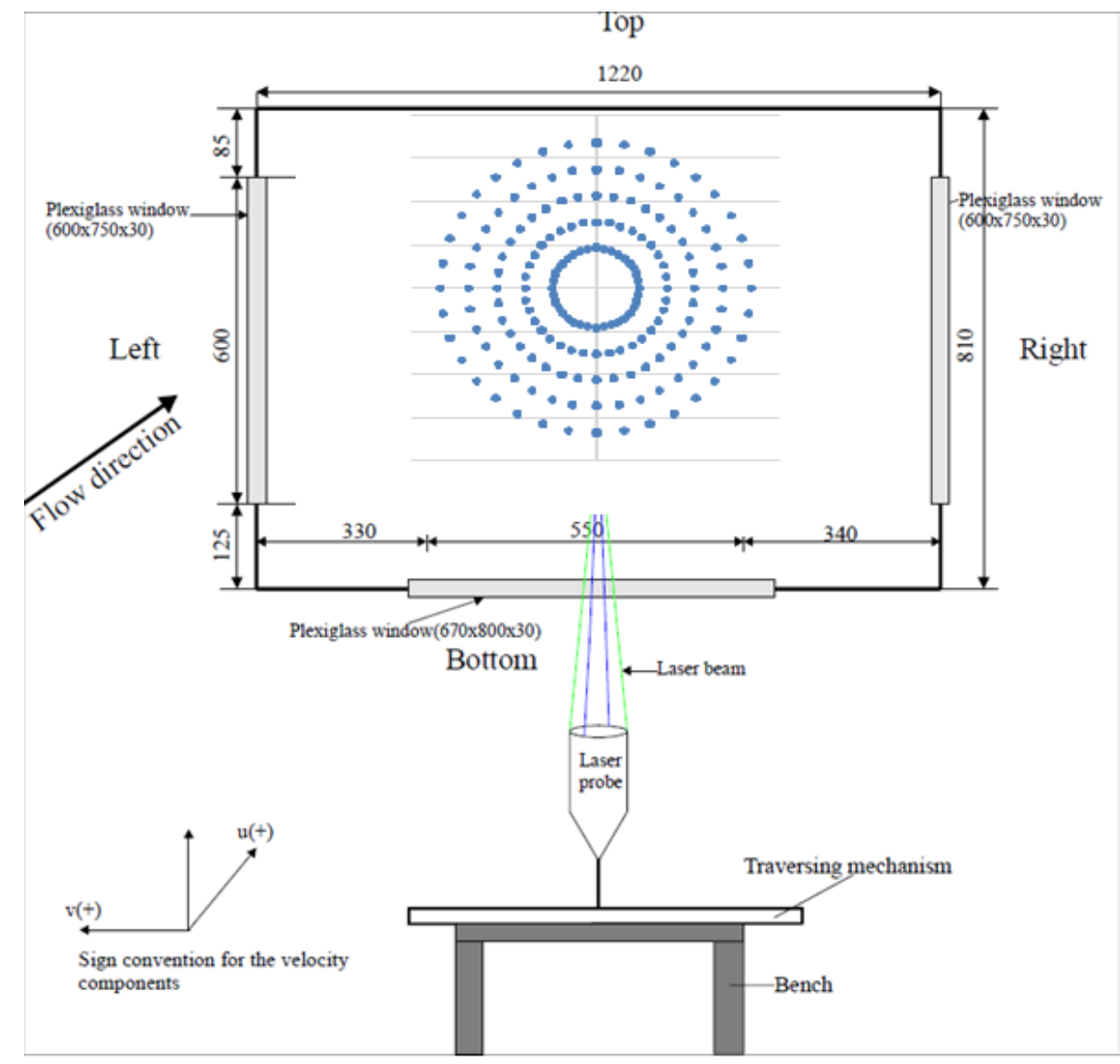

Figure 4 Grid of measurement points used for the wake screen development (reproduced from (Korkut, 1999)

The three wake screens manufactured and used for the experimental simulations are shown in Figure 5. During the experimental wake simulations these wake screens were placed ahead of the model propellers at a distance of 1.5 propeller diameters as shown in Figure 7 and wake velocity components were measured by using the ECT's two dimensional LDA system.

The simulations of the target wake velocities by using the final grid arrangements shown in Figure 5 were achieved by 20 attempts in total for the three different wake variations. The measurements with the finalized wake grids, which are presented as contour plots, are shown in Figure 6 for the three representative wake flows. 


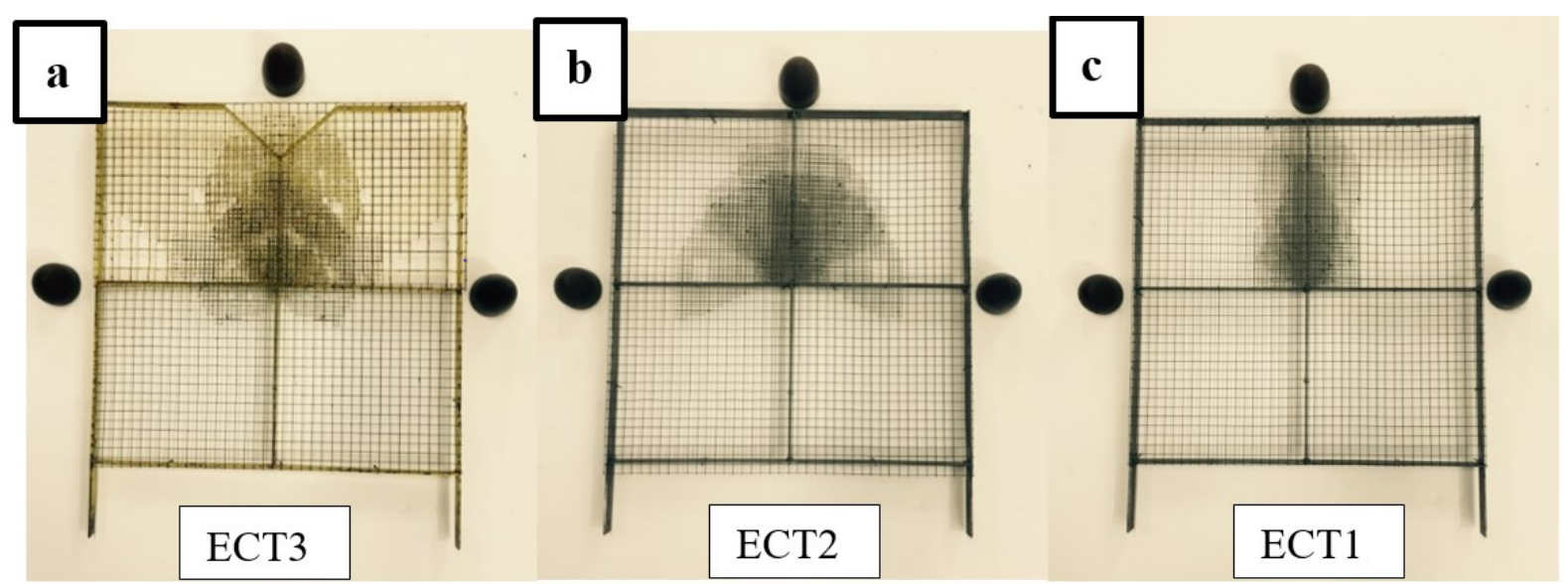

Figure 5 Constructed wake screens; ECT3 (a), ECT2 (b) and ECT1 (c).

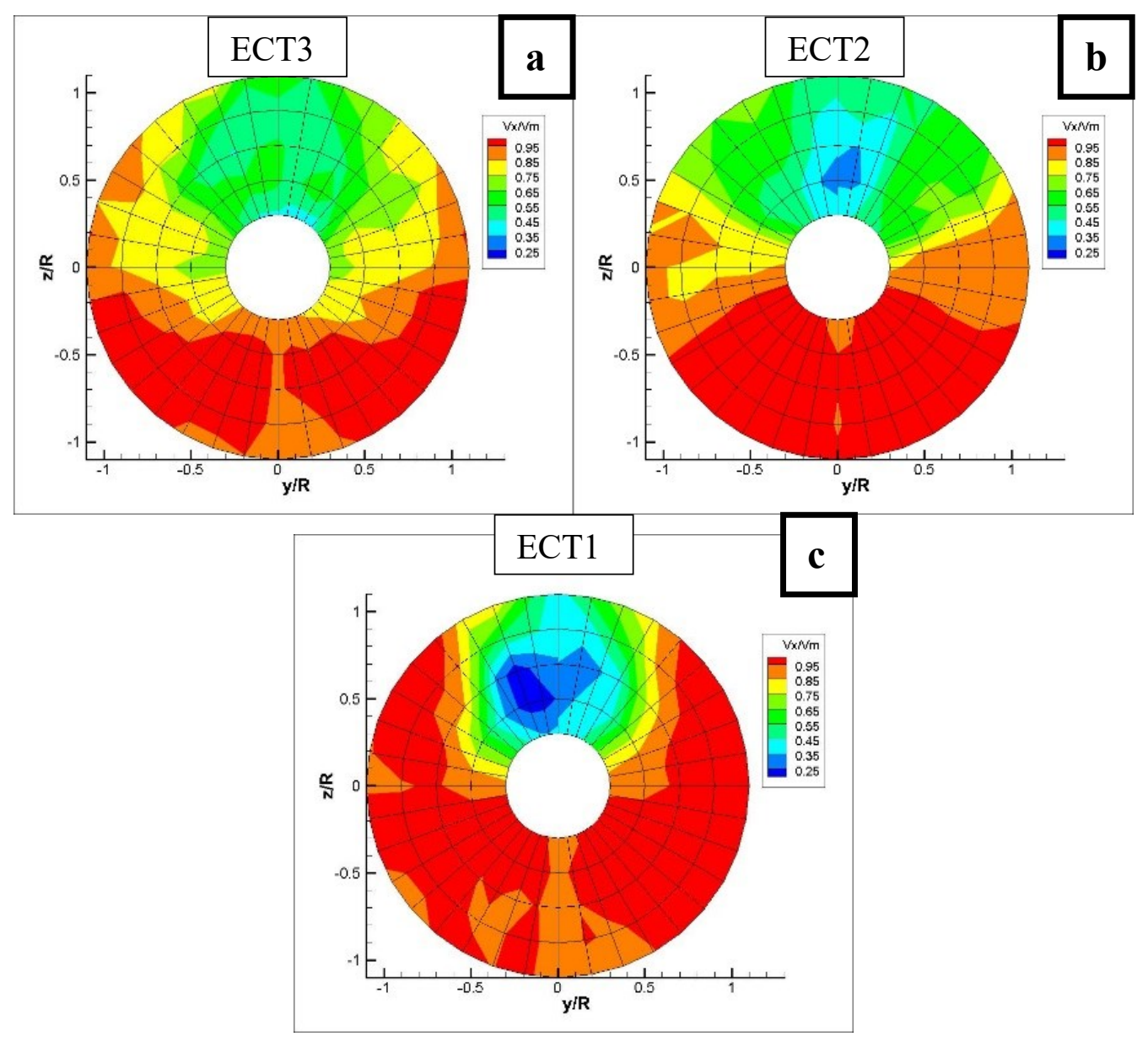

Figure 6 Contour plots of axial velocity distributions of the simulated representative wakes; ECT3 (a), ECT2 (b) and ECT1(c). 


\subsection{Experimental setup and test matrix}

In order to efficiently conduct the systematic propeller tests, an easy implementation and change of the experimental parameters was needed. In this context, in order to be able to acquire a large amount of data points, major variations to the experimental setup, such as the propeller and the wake screen, should require short periods of down time for the facility. The wake field can be generated for the experiments using the following configurations;

- Wire screen mesh for the cavitation tunnels with small test sections. Cost effective and relatively simple method simulating only axial velocity component of the wake field.

- "Dummy" models are utilized at medium size facilities in conjunction with wire screens as the removed parallel mid-section due to dummy model concept results in relatively lower Reynolds Number and hence a boundary layer which is not sufficiently developed

- Full ship models, which are used for the towing tank tests, are also used for the cavitation tunnel testing in the facilities with large test sections. There may still be a need for wake screens if the differences due to the extrapolation of the wake to full scale are large.

However, such configurations require significant amount of setting up time. Therefore, to simplify the experimental setup and reduce the down-time, three wake screens, presented in Section 2.2, have been used. The wake screens are located in the tunnel at a distance of 1.5 propeller diameter upstream of the propeller model in order to avoid the high levels of turbulence intensities introduced by the presence of the wake screen, and installed as seen in Figure 7 . The average turbulent intensity values for the open water conditions is $1.7 \%$ and $8.93 \%, 7.87 \%$ and $7.92 \%$ for ECT1, ECT2 and ECT3 respectively. 


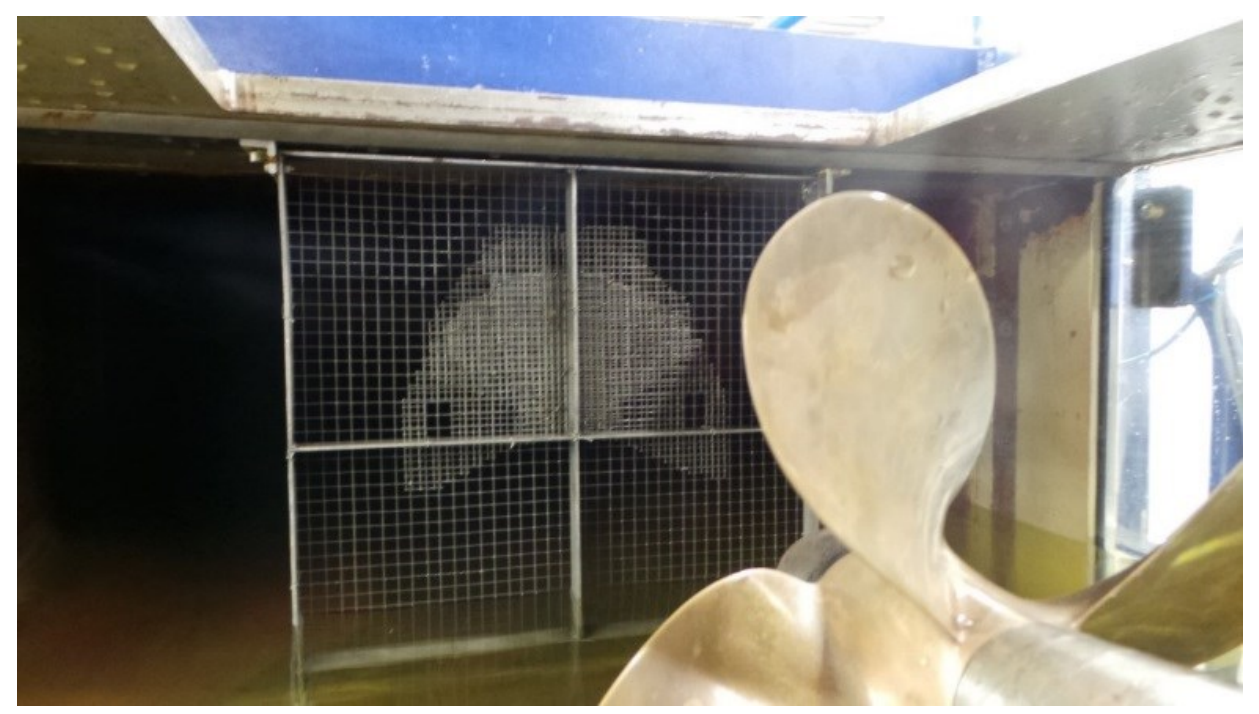

Figure 7 Testing set-up of model propeller behind wake ECT2 at a distance of 1.5 propeller diameter

Figure 8 shows the experimental set-up used in ECT including propeller model, Brüel \& Kjær (B\&K) 8103 miniature hydrophone and pressure sensor location on the top plate. Pressure sensor was located at approximately $35 \%$ of the propeller diameter above the propeller.

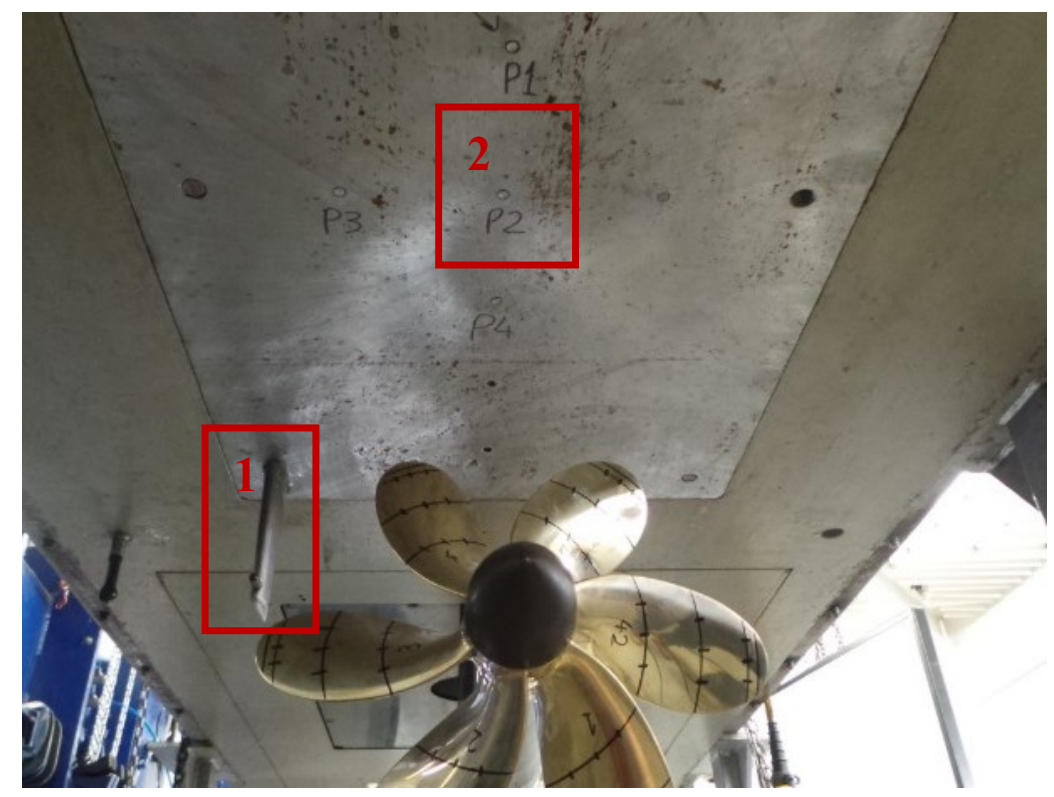

Figure 8 Experimental setup in ECT test section with hydrophone (1) and pressure sensor (2).

Six members of the Meridian series were hand-picked to assess key propeller design parameters; models KCD 191,192 and 193 were chosen to assess the effect of the pitch, while models KCD 129 and KCD 65 to assess the effect of blade number. Models KCD 65 and KCD 74 were selected to assess the effect of the BAR. Table 3 presents the variation of the propeller design 
parameters, whilst Figure 9 shows the propellers ready for the tests after marking with radius lines and chord length divisions. The same figure also includes the three wake screens used.

Table 3 Propeller design parameters of the chosen standard series subset

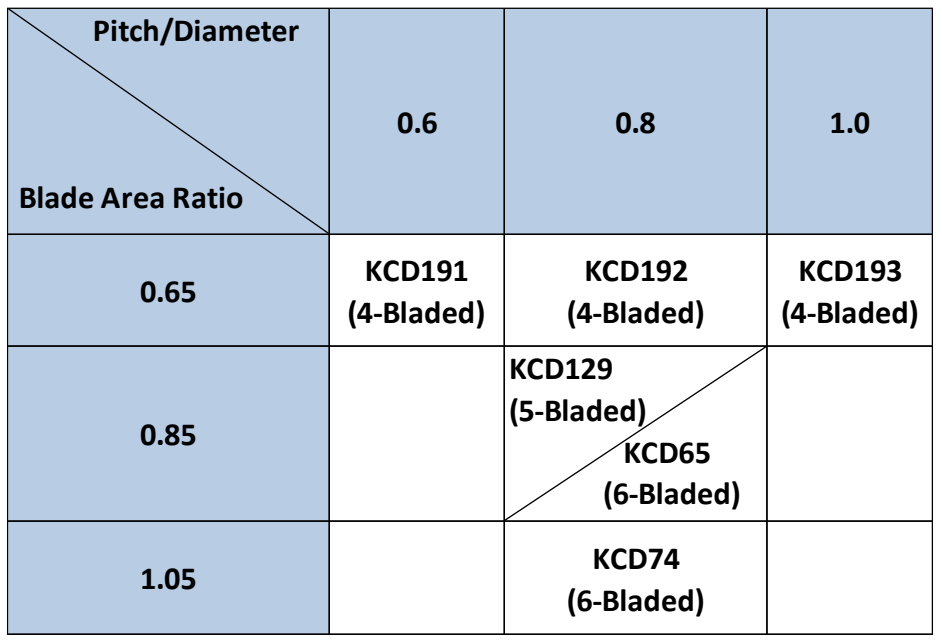




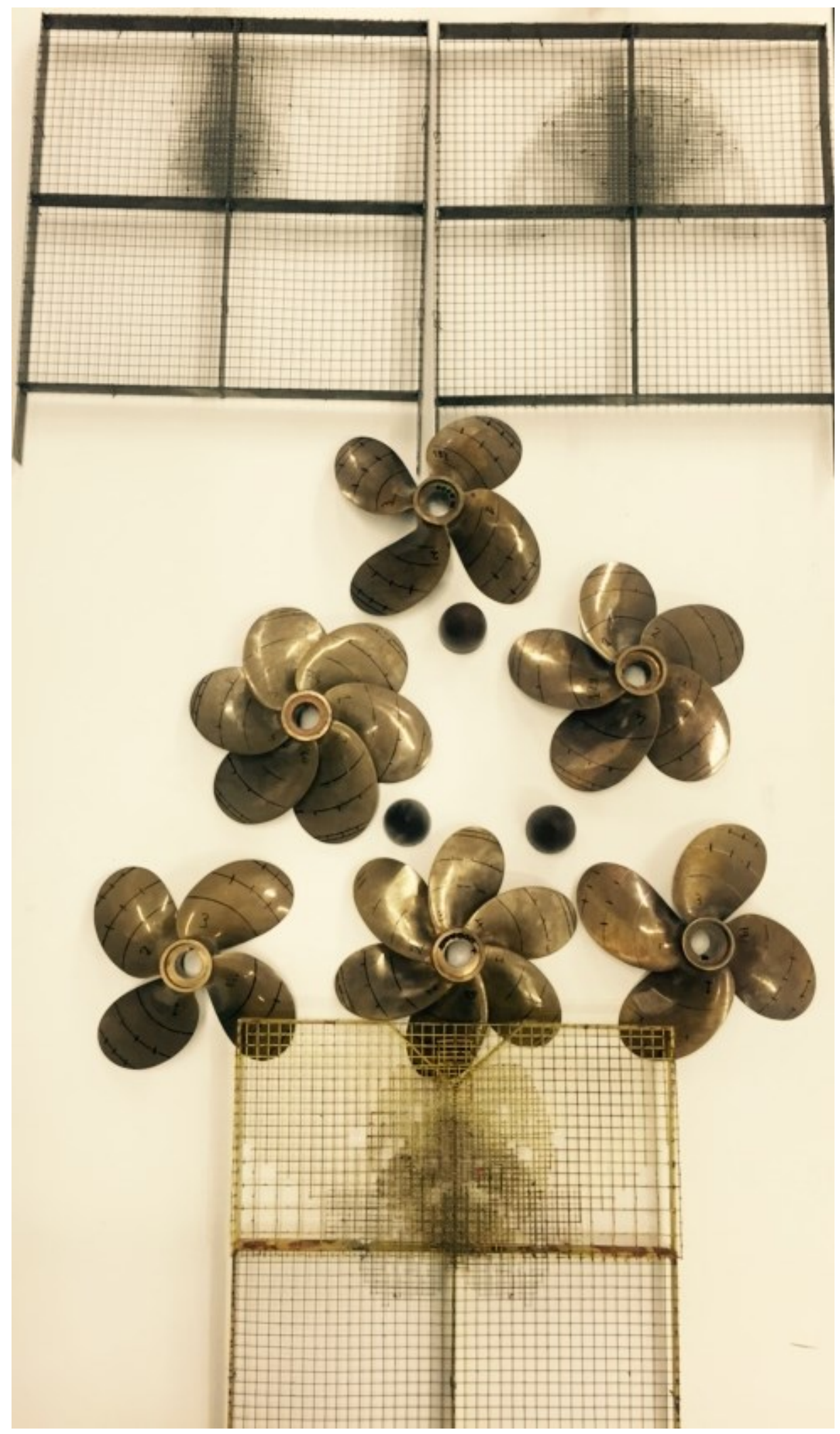

Figure 9 Meridian propellers and wake screens used for tests 
The acquired data were analysed using the well-known propeller performance parameters of advance coefficient $(\mathrm{J})$, thrust and torque coefficient $\left(\mathrm{K}_{\mathrm{T}}, 10 \mathrm{~K}_{\mathrm{Q}}\right)$ and propeller efficiency $\left(\eta_{0}\right)$, which are calculated using the formulations given by Equation 3.

$$
10 K_{Q}=\frac{10 Q}{\rho n^{2} D^{5}}, J=\frac{V_{A}}{n D}, K_{T}=\frac{T}{\rho n^{2} D^{4}}, \eta_{0}=\frac{J K_{T}}{2 \pi K_{Q}}
$$

Equation 3

Amongst various cavitation number formulations, the resultant cavitation number $\left(\sigma_{\mathrm{vr}}\right)$ takes the operational conditions (i.e. inflow velocity and rotational speed) better into account than more simple formulations. The calculation includes terms for both rotational speed of the propeller as well as the axial velocity component as in Equation 4.

$$
\sigma_{v r}=\frac{P_{a}+\rho g h_{s}-P_{v}}{0.5 \rho\left[(V)^{2}+(0.7 \pi D n)^{2}\right]}
$$

Equation 4

The systematic experimental data consisted of 576 individual tests, based on the selected six model propellers, conducted in uniform flow and behind the earlier described three systematically varied wake grids together with three different levels of tunnel vacuum conditions and eight different propeller speeds. A summary of the test matrix is shown in Table 4. The complete set of the conducted tests as a function of $\mathrm{J}$ against $\sigma_{\mathrm{vr}}, \mathrm{K}_{\mathrm{T}}, 10 \mathrm{~K}_{\mathrm{Q}}$ coefficients are presented by Figure 10. As can be seen from Figure 10, experimental test conditions are concentrated in the low cavitation number areas to ensure that the majority of the cases experience cavitation as it is the dominant noise source.

\begin{tabular}{|c|c|c|c|c|c|c|c|}
\hline $\begin{array}{c}\text { Model } \\
\text { propelle } \\
\text { r No } \\
\end{array}$ & $\mathbf{P} / \mathbf{D}$ & BAR & $\begin{array}{l}\text { Blade } \\
\text { no Z }\end{array}$ & $\begin{array}{l}\text { Wake flow } \\
\text { condition }\end{array}$ & $\begin{array}{l}\text { Vacuum } \\
\text { condition }\end{array}$ & $\begin{array}{c}\text { Shaft speed N } \\
\text { (rpm) }\end{array}$ & $\begin{array}{c}\text { Inflow } \\
\text { speed } \\
\text { V }(\mathrm{m} / \mathrm{s})\end{array}$ \\
\hline KCD 191 & 0.6 & 0.65 & 4 & \multirow{6}{*}{$\begin{array}{c}\text { Open water, } \\
\text { "ECT1", } \\
\text { "ECT2", "ECT3" }\end{array}$} & \multirow{6}{*}{$\begin{array}{c}\text { Atmospheri } \\
\text { c, } 150 \\
\mathrm{mmHg}, 300 \\
\mathrm{mmHg}\end{array}$} & \multirow{6}{*}{$\begin{array}{c}600,800 \\
1000,1200, \\
1400,1500, \\
1750,2000\end{array}$} & \multirow{6}{*}{3} \\
\hline KCD 192 & 0.8 & 0.65 & 4 & & & & \\
\hline KCD 193 & 1.0 & 0.65 & 4 & & & & \\
\hline KCD 129 & 0.8 & 0.85 & 5 & & & & \\
\hline KCD 65 & 0.8 & 0.85 & 6 & & & & \\
\hline KCD 74 & 0.8 & 1.05 & 6 & & & & \\
\hline
\end{tabular}

Table 4 Meridian series propeller cavitation noise test matrix 


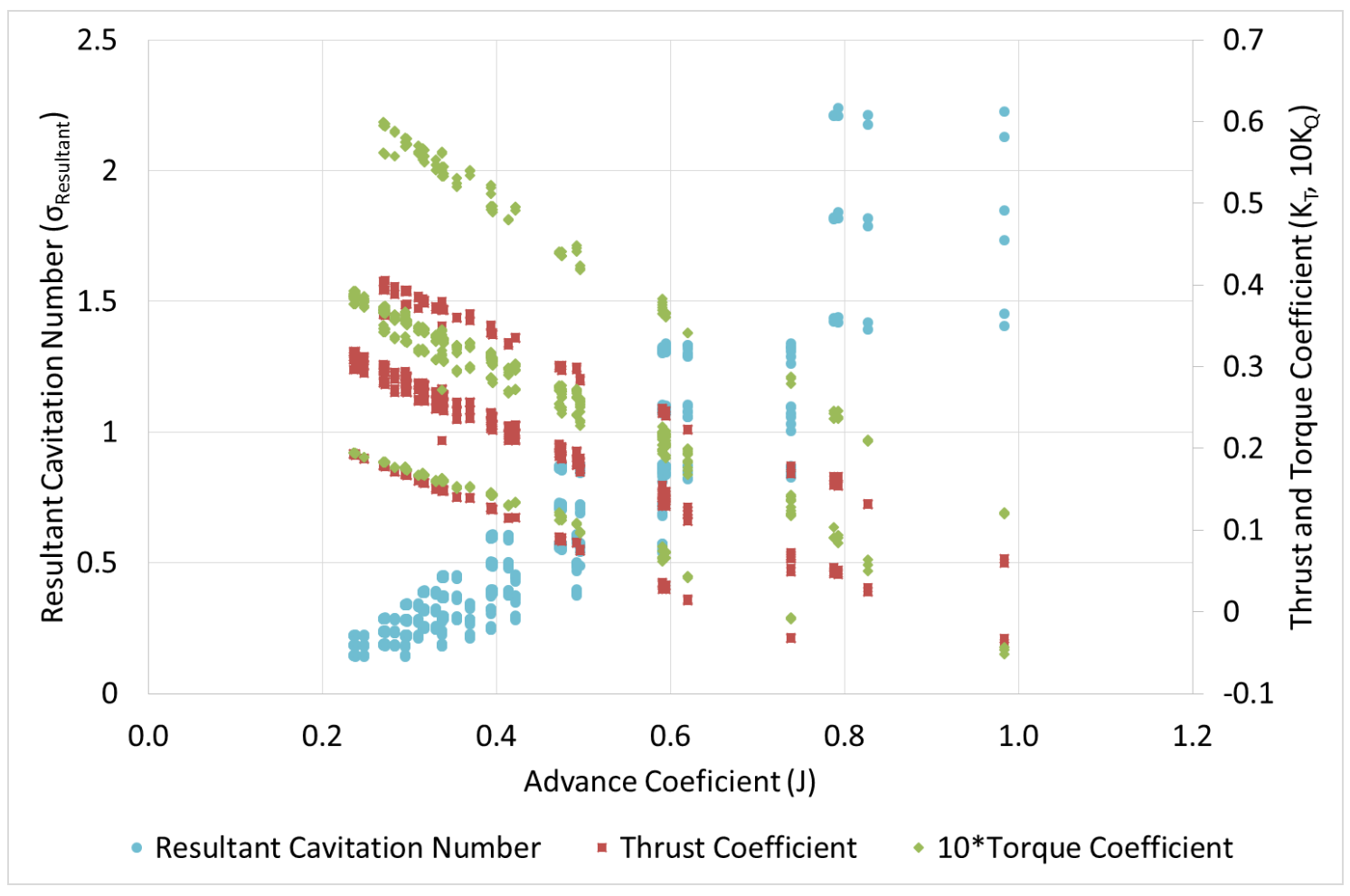

Figure 10 Dataset illustration as a function of advance coefficient $(J)$ in conjunction with resultant cavitation number $\left(\sigma_{\text {Resultant }}\right)$, thrust $\left(K_{T}\right)$ and torque $\left(10 K_{Q}\right)$ coefficients

The water quality of the ECT is regulated throughout all cavitation tests as it has a significant impact on the measurements. It has been brought to the levels suggested by the ITTC (ITTC, 2011, 1987). ECT has a dedicated water quality monitoring system and degassing system to keep the oxygen saturation level of the tunnel at desired level. The tunnel water quality is recorded using two systems in conjunction. The first device enables continuous monitoring of the water quality using the MS5 mini sonde and the dedicated Hydrolab software. Oxygen saturation is calculated as the percentage of dissolved $\mathrm{O}_{2}$ concentration relative to that when completely saturated at the temperature of the measurement depth. The probes attached to the tip of the mini sonde measure the water quality within a tubing arrangement through which tunnel water is pumped. The second device is a hand held meter for dissolved oxygen, from YSI instruments. This enables instantaneous on demand measurements of the water quality for cross checking with the continuous monitoring system.

The tunnel was deaerated by means of its dedicated degassing system as required. The dissolved oxygen content was increased by using the degassing cylinders under atmospheric condition and by circulating the tunnel for long time periods to enable saturation of the gas in 
the tunnel water. The tunnel dissolved oxygen content was kept between 30 to $40 \%$ throughout the periods that experiments took place following the ITTC guidelines.

\section{Presentation of results and advance analysis of cavitation- induced noise data using JTFA technique}

Traditionally URN measurements are presented in the frequency domain thus enabling the comparisons of the URN levels as an indicator of the relative noise performance of a propeller. However, this approach limits the interpretation of various causes for the generation of URN as well as understanding the detailed effects of these causes. In order to provide further insight into this matter an advanced analysis tool for the cavitation-induced URN is discussed in this section. The implementation of this tool has required the recordings of the pressure pulses at a strategic location above the tested propellers in synchronisation with the cavitation observations using High-Speed Video (HSV) cameras during the course of the systematic tests and complementing noise measurements with hydrophones. The synchronization was achieved via Transistor-Transistor Logic (TTL) signal from the electric motor driving the shaft to trigger both the pressure sensor's (MEAS XPM10-S107-05BS pressure sensors) Data Acquisition (DAQ) system and the high-speed video. Both the pressure pulses and the cavitation observations were acquired at a rate of $5000 \mathrm{~Hz}$ to allow a fine assessment of the time signal with respect to the visual cavitation phenomena observed, as presented by Figure 11.

Within the framework of the above approach, this section is comprised of three analysis phases. The first phase presents the analysed net noise levels in the frequency domain for the chosen sample data and is discussed in Section 3.1. Following this, the synchronized time signals and cavitation observations are put under the spotlight in the second phase presented in section 3.2. Finally, the raw pressure pulse signals are processed by using the Joint Time-Frequency Analysis (JTFA) toolkit of LabVIEW to enable the determination of the frequency band to which certain types of cavitation phenomena contribute. This is presented and discussed in Section 3.3. An overall presentation of this advanced tool for the analysis of the cavitationinduced URN and background is outlined in Figure 12. 


\section{Signal Conditioner}

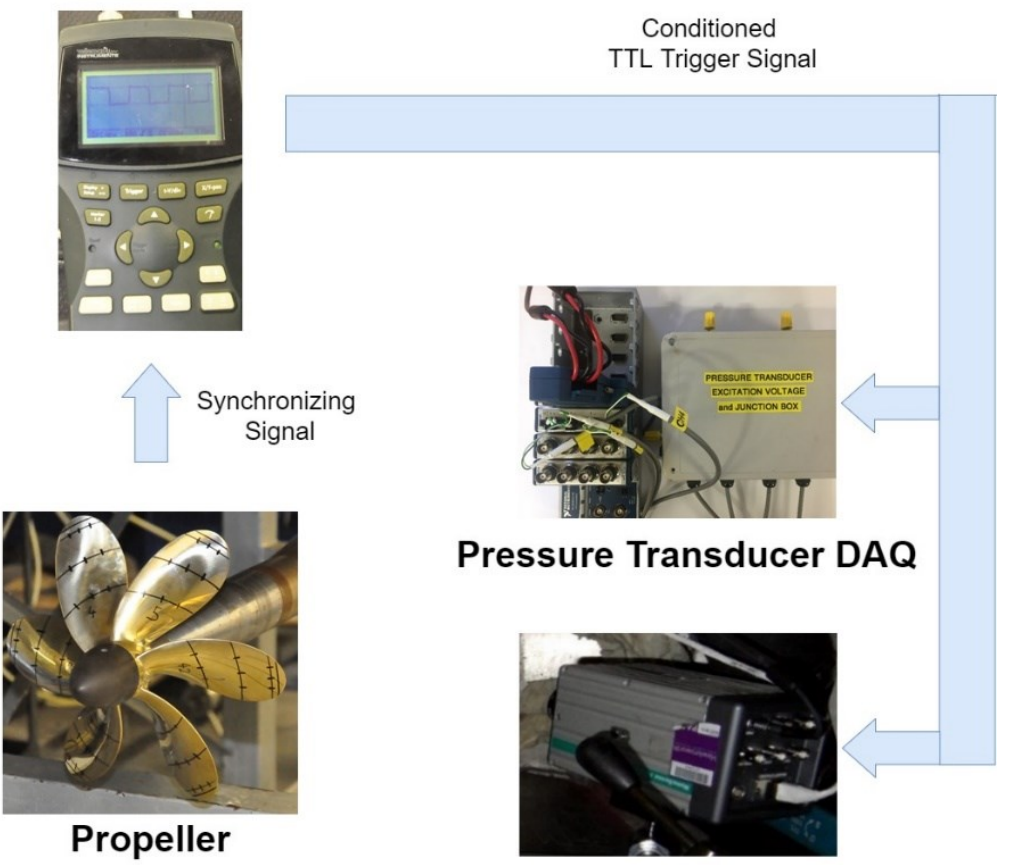

High-Speed Camera

Figure 11 Elements of synchronized pressure pulse and cavitation observation system

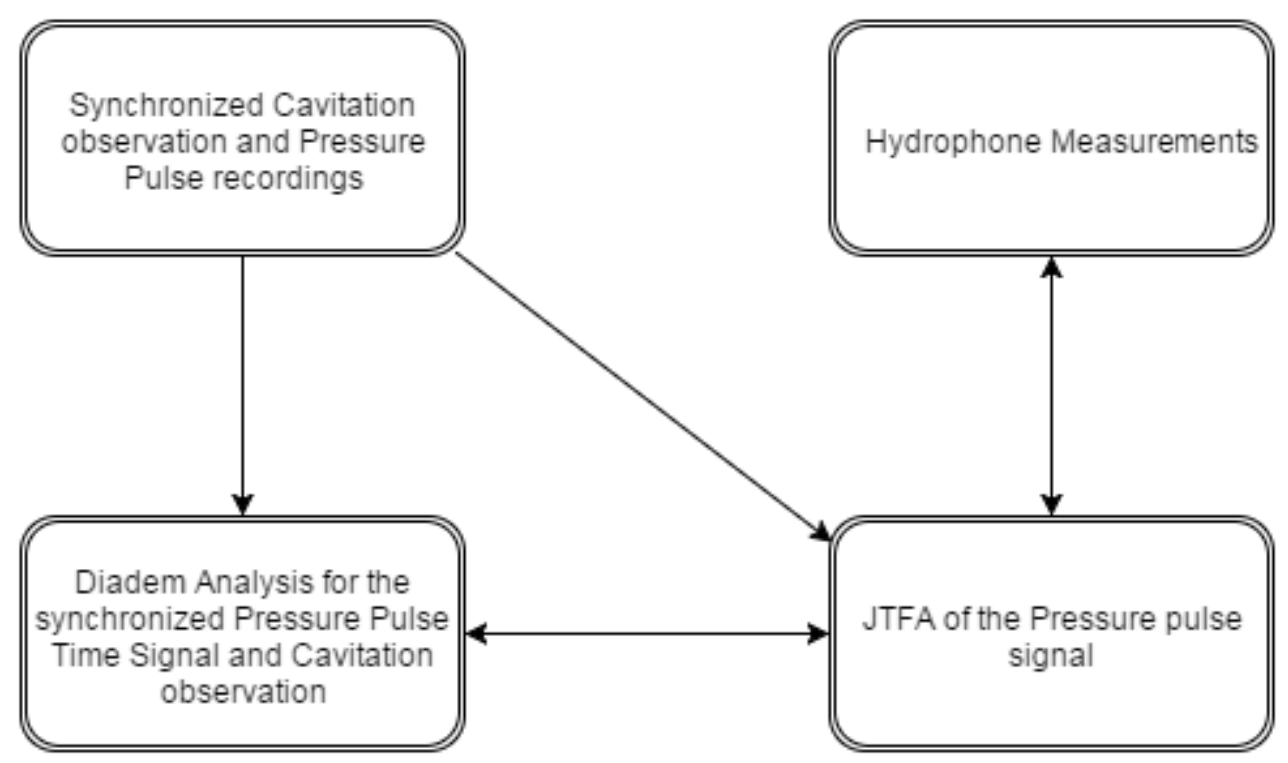

Figure 12 Layout of advance analysis tool for cavitation induced URN.

National Instruments DIAdem and LabVIEW software tools are used to quickly locate, load, visualize, analyse, and report the measurement data collected during the data acquisition and/or generated during simulations (National Instruments, 2011, 2003). This tool kit is designed to meet the demands of today's testing environments, which require quick access, processing, and 
reporting on large volumes of scattered data in multiple custom formats to make informed decisions.

\subsection{URN data of propellers in frequency domain}

The noise measurements are conducted using a B\&K 8103 hydrophone mounted within the tunnel in a streamlined strut. The hydrophone is positioned facing the incoming flow and at an offset of $380 \mathrm{~mm}$ as shown in Figure $13(x=264 \mathrm{~mm}, y=245 \mathrm{~mm} . \mathrm{z}=120 \mathrm{~mm})$. The inherent selfnoise experienced by the propeller is offset by background noise correction.

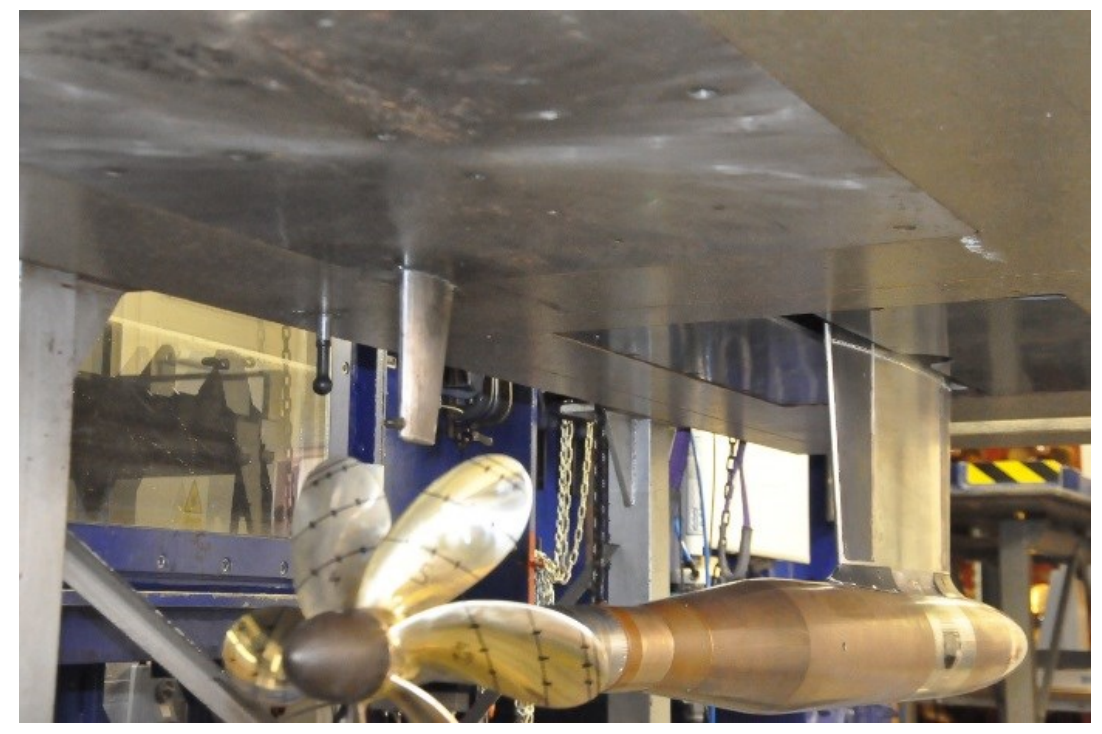

Figure 13 Location of B\&K hydrophone relative to model propeller inside ECT

The noise data acquisition for 1/3-octave band was conducted by using the waterfall format of the $\mathrm{B} \& \mathrm{~K}$ noise analysis software in order to eliminate the effect of any instantaneous effects/noise (i.e. any external noise that may influence the measurement). This was achieved by using the multi-buffer option of the software which triggers the system every 0.25 seconds for the next measurement. A total of 200 triggers or 50 seconds was measured per condition, each at a $45 \mathrm{kHz}$ sampling rate. The measured values are corrected to an equivalent $1 \mathrm{~Hz}$ bandwidth and $1 \mathrm{~m}$ source level. A common practice in the analysis and presentation of the noise levels is to reduce the measured values of Sound Pressure Level (SPL) in each 1/3-octave band to an equivalent $1 \mathrm{~Hz}$ bandwidth by means of the correction formula recommended by ITTC (1987) as in Equation 5.

$$
S P L_{1}=S P L_{m}-10 \log _{10}(\Delta f)
$$


Where; $\mathrm{SPL}_{1}$ is the sound pressure level in $1 \mathrm{~Hz}$ bandwidth in $\mathrm{dB}$ relative to $1 \mu \mathrm{Pa}$. $\mathrm{SPL}_{\mathrm{m}}$ is the sound pressure level in $1 / 3$-octave bandwidth in $\mathrm{dB}$ relative to $1 \mu \mathrm{Pa}$. $\Delta \mathrm{f}$ is the frequency bandwidth for each respective 1/3-octave band.

The ITTC also requires that the sound pressure levels are corrected to a standard measuring distance of $1 \mathrm{~m}$ using the spherical acoustic wave propagation relationship using Equation 6.

$$
S P L=S P L_{1}+20 \log _{10}\left(R_{A}\right) \quad \text { Equation } 6
$$

where; SPL is the Sound Pressure Level in $1 \mathrm{~Hz}$ band in $\mathrm{dB}$ relative to $1 \mu \mathrm{Pa}$ at $1 \mathrm{~m}$. $\mathrm{r}_{\text {acoustic }}$ is the distance of the location of the hydrophone from the propeller centre as it is assumed to be the location of the acoustic source.

Transfer functions for the ECT have been determined for the systematic propeller cavitation tests. The resulting reverberation characteristics of the ECT has been observed to be significantly similar to other cavitation testing facilities (Tani et al., 2016). The conducted study is not applied as a correction to the experimental measurements carried out in the context of this section due to the limited frequency range for which the signal-to-noise ratio of the measurements is sufficient (only corresponding to three $1 / 3$-octave centre frequencies). In order to overcome this missing information, further investigations are planned to take place using a hydrophone array to enable the spatial averaging of the cavitation tunnel measurements.

The corrections for the tunnel background noise measurements are applied to the measured total noise levels in order to determine the net cavitation noise. The background noise measurements are made without the propeller model, which was replaced by a dummy hub (Bretschneider et al., 2014). The background noise correction applied depending on the level of the difference following the procedure in ANSI/ASA S12.64-2009/Part1. When the difference is smaller than $3 \mathrm{~dB}$, the result is discarded. In case of a difference between 3 and $10 \mathrm{~dB}$, the results are corrected according to Equation 7 and no correction is applied in case of the difference being greater than $10 \mathrm{~dB}$.

$$
\left.\left.S P L_{N}=10 \log \left[10^{\left(S P L_{T} / 10\right.}\right)-10^{\left(S P L_{B} / 10\right.}\right)\right]
$$

where subscripts $N, T$ and $B$ indicate net, total and background respectively. 
Figure 14 to Figure 18 illustrate the influence of various propeller parameters on URN, as well as the effect of wake inflow and operating conditions. Sequentially, Figure 14 shows the effect of thrust coefficient; Figure 15 demonstrates the effect of the wake inflow; Figure 16 shows the influence of the blade number; Figure 17 depicts the influence of the BAR and Figure 18 illustrates the effect of the $\mathrm{P} / \mathrm{D}$ ratio.

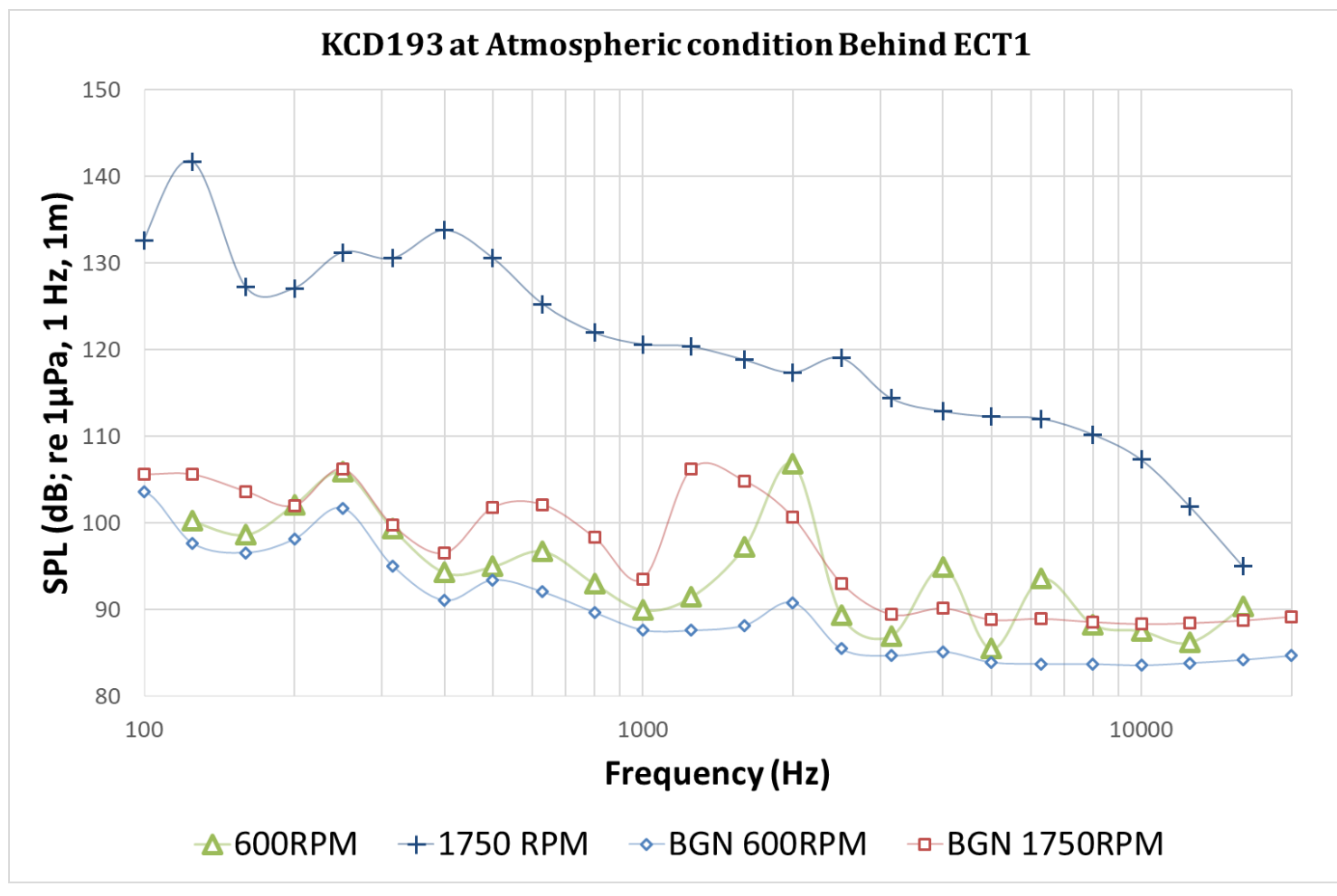

\begin{tabular}{|c|c|c|c|c|c|c|c|c|c|}
\hline $\begin{array}{c}\text { Propeller } \\
\text { No }\end{array}$ & P/D & Ae/Ao & $\begin{array}{c}\text { Blade } \\
\text { no. } \mathbf{Z}\end{array}$ & Wake & $\mathbf{J}$ & $\mathbf{K}_{\mathbf{T}}$ & $\mathbf{1 0 K} \mathbf{Q}$ & $\mathbf{N}$ & $\boldsymbol{\sigma}_{\mathbf{v r}}$ \\
\hline KCD193 & 1 & 0.65 & 4 & ECT1 & 0.82 & 0.13 & 0.21 & 605 & 2.21 \\
\cline { 5 - 9 } & & & 0.28 & 0.40 & 0.59 & 1751 & 0.29 \\
\hline
\end{tabular}

Figure 14 The comparison of the KCD193 propeller behind ECT 1 wake at propeller rotational speed of 600 RPM and 1750 RPM together with the corressponding Background Noise levels denoted by BGN.

Figure 14 presents the impact of the cavitation on the measured SPLs caused by an increase in the shaft speed and resulting change in the shaft speed-based cavitation numbers and propeller loading. The figure clearly shows that the SPLs present a significant increase over the whole frequency range with amplitudes over $30 \mathrm{~dB}$ higher. Although such an observation is expected to be obvious, this also supports the hypothesis of the cavitation being the dominant noise source once it develops. Figure 14 also presents the background noise levels measured at these conditions denoted with BGN for both conditions. 


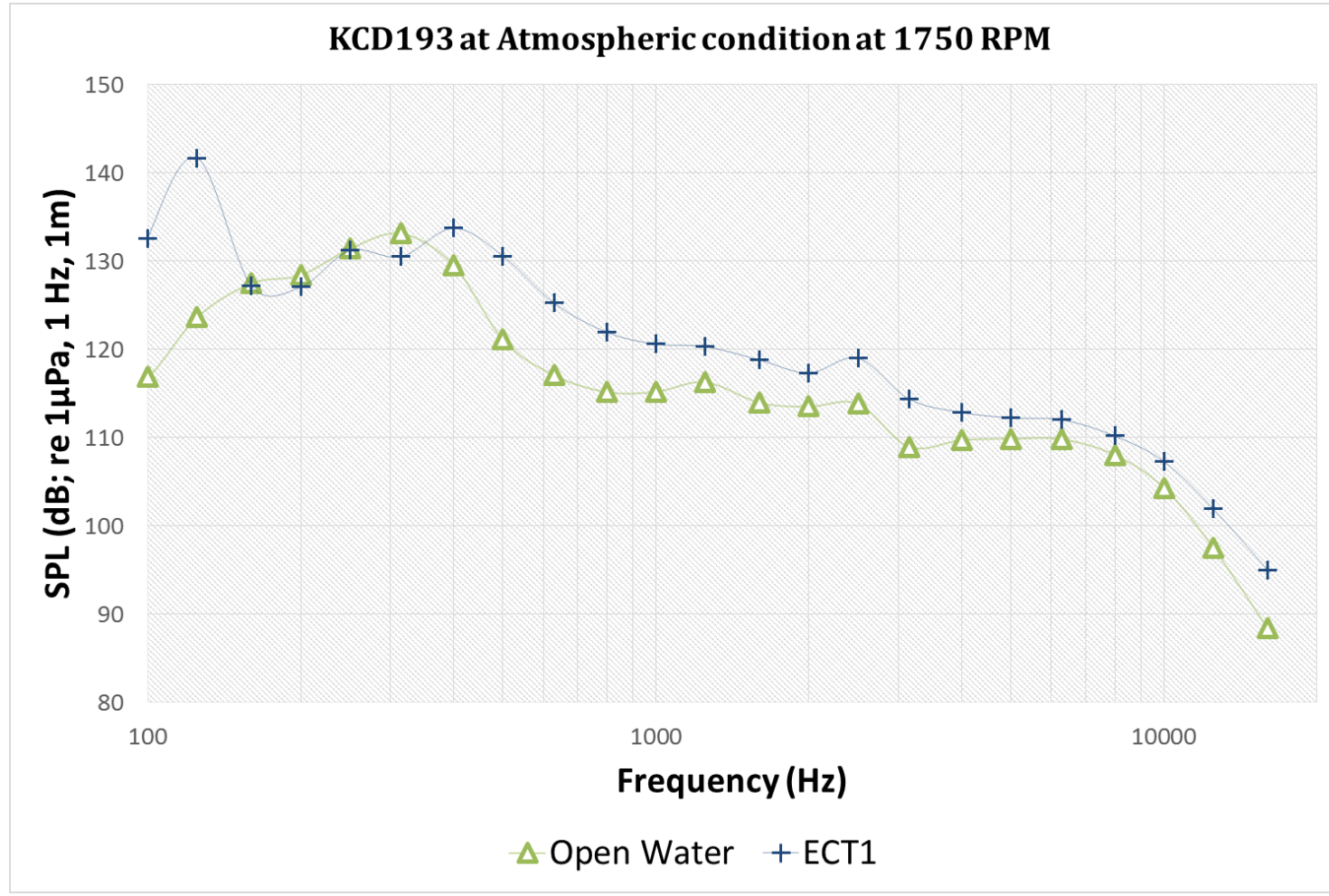

\begin{tabular}{|c|c|c|c|c|c|c|c|c|c|}
\hline $\begin{array}{c}\text { Propeller } \\
\text { No }\end{array}$ & $\mathbf{P} / \mathbf{D}$ & Ae/Ao & $\mathbf{Z}$ & Wake & $\mathbf{J}$ & $\mathbf{K}_{\mathbf{T}}$ & $\mathbf{1 0 K}_{\mathbf{Q}}$ & $\mathbf{N}$ & $\boldsymbol{\sigma}_{\mathbf{v r}}$ \\
\hline KCD193 & 1 & 0.65 & 4 & ECT1 & 0.28 & 0.40 & 0.59 & 1751 & 0.29 \\
\cline { 5 - 10 } & & Uniform & 0.34 & 0.38 & 0.56 & 1752 & 0.29 \\
\hline
\end{tabular}

Figure 15 Noise comparison of the KCD193 propeller behind ECT 1 and in open water condition at propeller speed of 1750 RPM

Figure 15 provides further evidence on the claim that the cavitation developed behind a nonuniform wake may induce higher SPLs, which can be up to $20 \mathrm{~dB}$ higher at the $1^{\text {st }}$ blade passage frequency (BPF) and no less than $5 \mathrm{~dB}$ difference at the broadband frequencies, compared to the SPLs measured in open water condition. This indicates that although the cavitation is the dominating factor for the URN, the dynamics involved due to the cavitation volume acceleration caused by the wake variation causes further elevation that may also be contributed by bursting, rebounding and collapse of the cavitation bubbles. Such cavitation dynamics mean that there is an acceleration of the cavitation volume, which is well known to be the main factor behind the hydro-acoustic cavitation noise. 


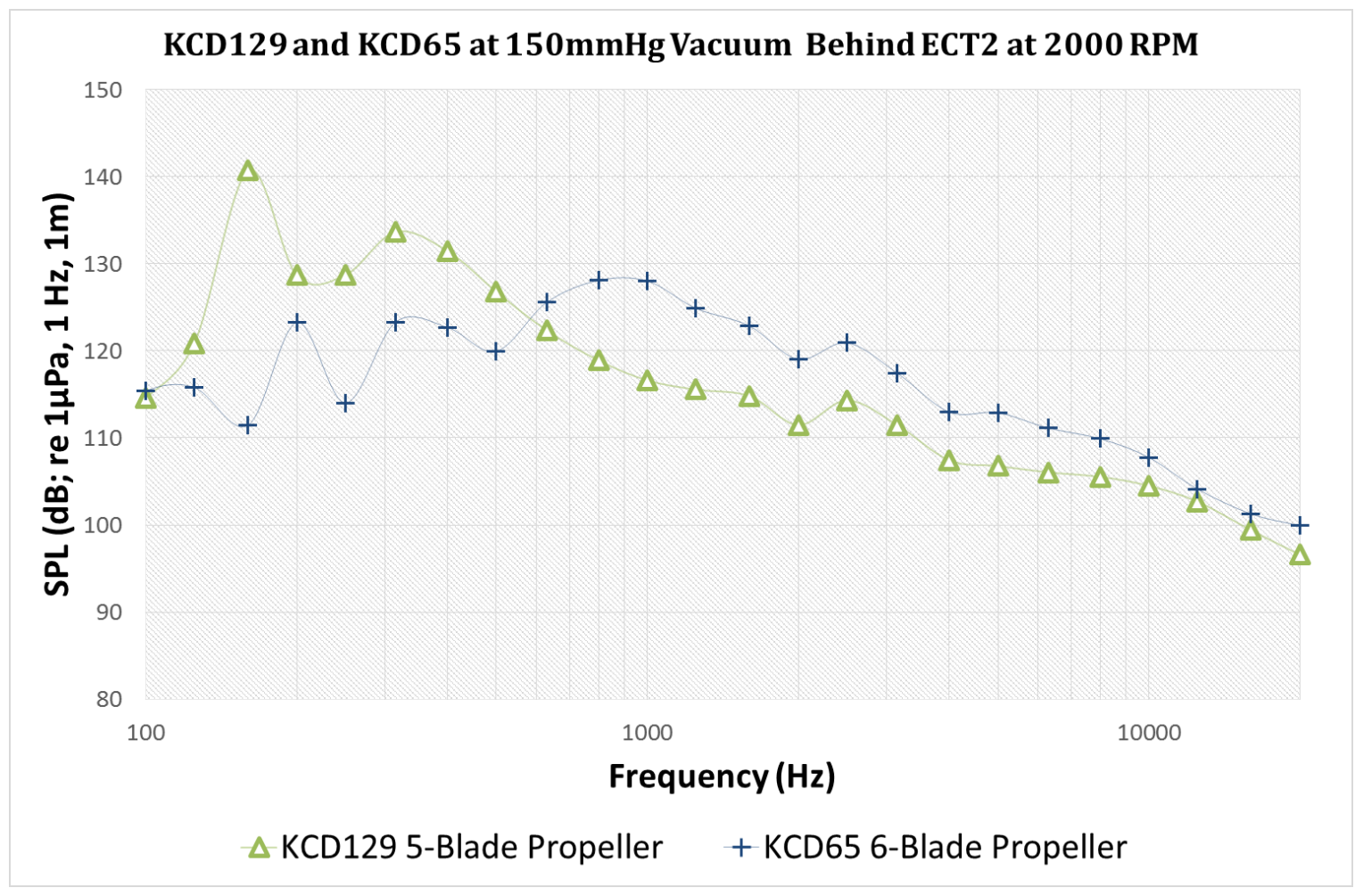

\begin{tabular}{|c|c|c|c|c|c|c|c|c|c|}
\hline $\begin{array}{c}\text { Propeller } \\
\text { No }\end{array}$ & P/D & BAR & $\begin{array}{c}\text { Blade } \\
\text { No }\end{array}$ & Wake & J & $\mathbf{K}_{\mathbf{T}}$ & $\mathbf{1 0 K}_{\mathbf{Q}}$ & $\mathbf{R P M}$ & $\boldsymbol{\sigma}_{\mathbf{v r}}$ \\
\cline { 1 - 5 } KCD129 & \multirow{2}{*}{0.8} & 0.85 & 5 & \multirow{2}{*}{ ECT2 } & 0.24 & 0.32 & 0.39 & 1992 & \multirow{2}{*}{0.18} \\
\cline { 1 - 1 } 65 & & 6 & & 0.31 & & 1990 & \\
\hline
\end{tabular}

Figure 16 Noise comparison of KCD 129 and KCD 65 propellers behind ECT 2 wake at propeller speed of 2000 RPM

Figure 16 shows differences due to the number of blades on the measured SPLs from two tested propellers. Interestingly, the spectral levels emitted by the two propellers cross over at a frequency around $600 \mathrm{~Hz}$. The KCD 129 (5-bladed) displays higher SPLs below the cross-over frequency while KCD 65 (6-bladed) emits higher SPLs above this frequency. While this will be dominated by the nature of the cavitation experienced by each propeller over the frequency range tested, the lesser blade number of KCD 129 will induce relatively higher thrust density per blade compared to KCD65. 


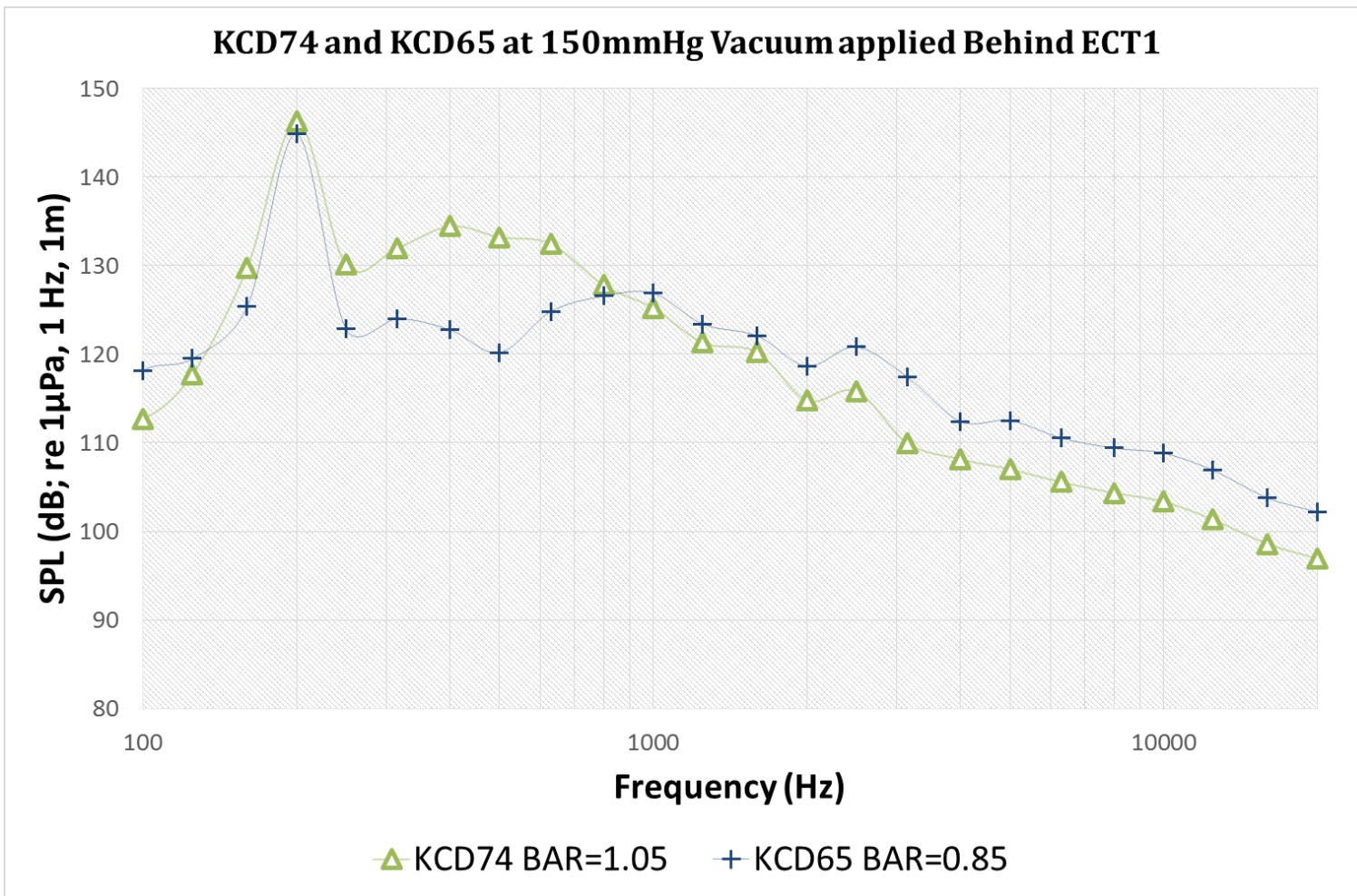

\begin{tabular}{|c|c|c|c|c|c|c|c|c|c|}
\hline $\begin{array}{c}\text { Propeller } \\
\text { No }\end{array}$ & $\mathbf{P} / \mathbf{D}$ & BAR & $\begin{array}{c}\text { Blade } \\
\text { No }\end{array}$ & V & $\mathbf{J}$ & $\mathbf{K}_{\mathbf{T}}$ & $10 K_{Q}$ & RPM & $\sigma_{\text {Resultant }}$ \\
\hline KCD 65 & \multirow{2}{*}{0.8} & 0.85 & & \multirow{2}{*}{ ECT1 } & \multirow{2}{*}{0.25} & \multirow{2}{*}{0.31} & \multirow{2}{*}{0.38} & 1988 & 0.19 \\
\hline KCD 74 & & 1.05 & & & & & & 1976 & 0.18 \\
\hline
\end{tabular}

Figure 17 The comparison of the KCD 74 and KCD 65 propellers behind ECT 1 wake at propeller speed of 2000 RPM

The comparison of the effect of the BAR is presented in Figure 17. The SPLs are observed to be similar up to $200 \mathrm{~Hz}$, which is the blade passage frequency. However, there seems to be a hump in the spectral levels experienced by the KCD 74 from $200 \mathrm{~Hz}$ to $800 \mathrm{~Hz}$ emitting higher sound pressure levels within this frequency range. KCD 65 is observed to produce higher noise levels for the high frequency range $(>800 \mathrm{~Hz})$ when compared to KCD 74 . This figure is intriguing as the propeller performance coefficients are exactly same and cavitation numbers are similar but there is significant difference in the URN. The impact of the BAR reveals itself in terms of the thrust per blade area. Thus, the heavily-loaded KCD 65 experiences more dynamic cavitation contributing to the high-frequency region of the measured spectra. 


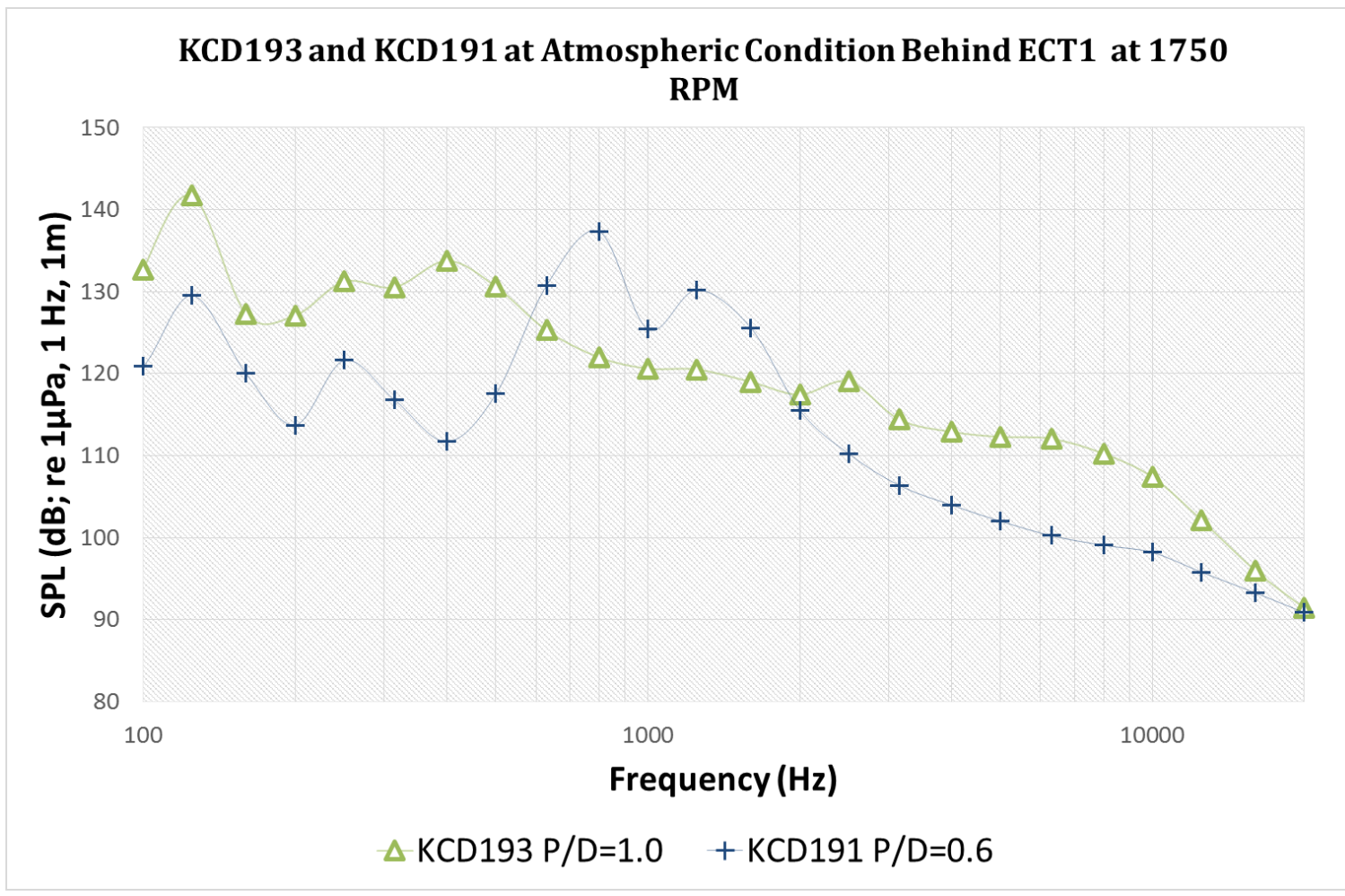

\begin{tabular}{|c|c|c|c|c|c|c|c|c|c|}
\hline $\begin{array}{c}\text { Propeller } \\
\text { No }\end{array}$ & P/D & BAR & $\begin{array}{c}\text { Blade } \\
\text { No }\end{array}$ & Wake & J & $\mathbf{K}_{\mathbf{T}}$ & $\mathbf{1 0 K} \mathbf{Q}$ & $\mathbf{R P M}$ & $\boldsymbol{\sigma}_{\text {Resultant }}$ \\
\cline { 1 - 6 } KCD193 & 1 & 0.65 & 4 & ECT1 & 0.28 & 0.40 & 0.59 & 1751 & 0.29 \\
\hline KCD191 & 0.6 & 0.17 & 0.18 & 0.29 \\
\hline
\end{tabular}

Figure 18 The comparison of the KCD 193 and KCD 191 propellers behind ECT 1 wake at propeller speed of 1750 RPM

The final comparison presented by Figure 18 is to represent the effect of different pitch ratios on the measured SPLs. While the noise levels of KCD193, which has a higher P/D and hence thrust loading, are generally higher than those of KCD 191 over the frequency range tested, this trend changes over a frequency band between $600 \mathrm{~Hz}$ to $2000 \mathrm{~Hz}$. Over this frequency band KCD 191, which has a lower P/D, displays two distinct humps that results in higher SPLs than for the KCD 193 SPLs. The reason behind the presence of the humps will be scrutinized further using the synchronized cavitation observation and pressure time signal method and JTFA in the following sections. As one may also note that the BPF levels also present more than $10 \mathrm{~dB}$ difference due to large sheet cavitation experienced by KCD193. 


\subsection{Synchronized pressure-fluctuation measurements and cavitation behaviour observations in the time domain}

These measurements were performed whilst capturing videos and pressure synchronously in an effort to relate cavitation images to characteristics of the measured pressure time-series. Synchronisation was achieved using a TTL signal from the motor speed encoder and a signal generator as a conditioner. The synchronized recordings and analysis of the results can enable one to make comparison of the time signals measured for each propeller to explore the effect of different propeller design and operational parameters on the URN with more detail. Within this framework, some of the earlier investigated parameters have been further explored based on the analysis of the synchronized pressure signals and cavitation observations and results thereof are discussed in the following paragraphs. The duplicated trigger signal was then fed into the high-speed video camera and pressure pulse DAQ system. Figure 19 shows the set-up at ECT used during the tests.

Nanosense high speed cameras were also used at $5000 \mathrm{fps}$ in a continuous recording mode for the non-uniform inflow conditions. A high intensity light source was obtained from PlasmaLite continuous light as shown in Figure 19, to allow a high acquisition rate for the high speed cameras. The measurements were performed whilst capturing videos and pressure synchronously in an effort to relate cavitation images to characteristics of the measured pressure time-series.

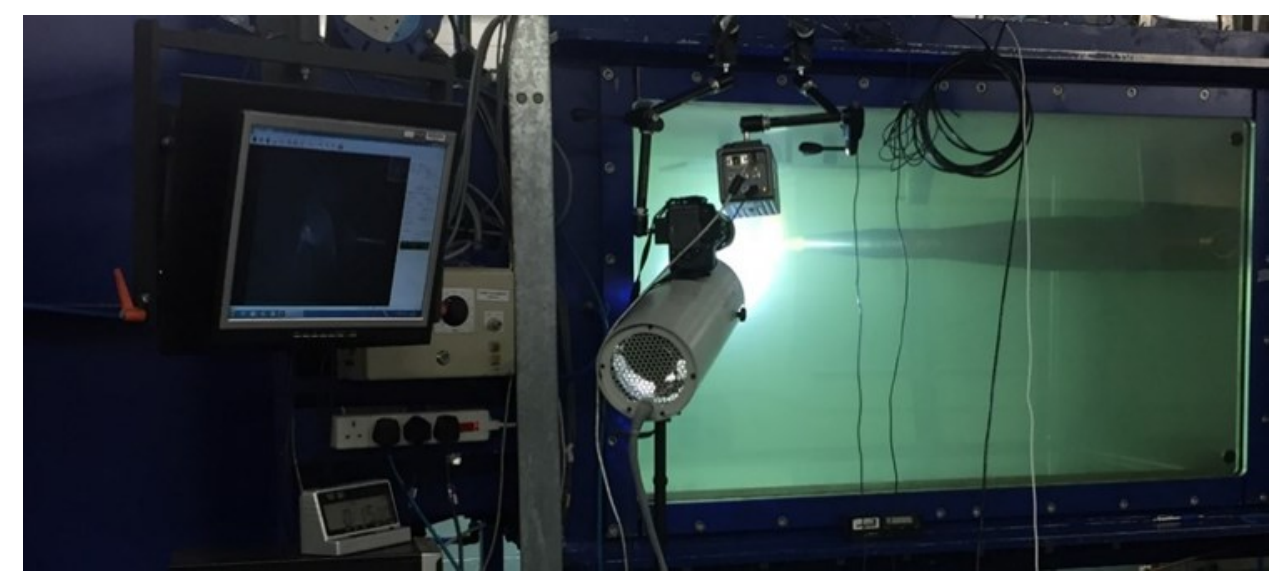

Figure 19 Model-scale high-speed video cavitation observation set-up at ECT

The pressure data from the sensor were acquired using the National Instruments (NI) DAQ system. The cDAQ 9178 chassis was used with the NI 9239 module for the signal output recording of the pressure sensors. The pressure sensors were powered using a laboratory direct 
current power supply being distributed to each sensor by means of a dedicated pressure transducer excitation voltage and junction box.

Figure 20 to Figure 24 have been reproduced using the recording made during the standard series propeller tests, using Diadem software. As the high-speed video recordings can occupy quite large amounts of hard disk space, they have been made for 0.1 second by totalling 500 individual images at an acquisition rate of $5000 \mathrm{~Hz}$. Therefore, although the pressure signals are recorded for 5 seconds, they have been trimmed to match the length of the high-speed video recording to enable synchronized viewing within the software. In addition to the Figure 20 to Figure 24, videos for the whole duration of the cavitation obsevrations for each condition is provided in Appendix A. The user interface for the videos in Appendix A is arranged to be divided into two sections, where the left-hand side of the figure belongs to one propeller within which the upper part is the pressure time signal and bottom half is the corresponsing cavitation observation. The right-hand side of the figure is likewise arranged to present a different propeller or condition for easy comparison. A marker traces the time signal for the corresponding cavitation observation aiding the interpretation. While screen shots from the post-processing tool are provided within the following Figure 20 to Figure 24, the videos of the synchronized pressure pulse time signals and cavitation observations are provided within Appendix A. 

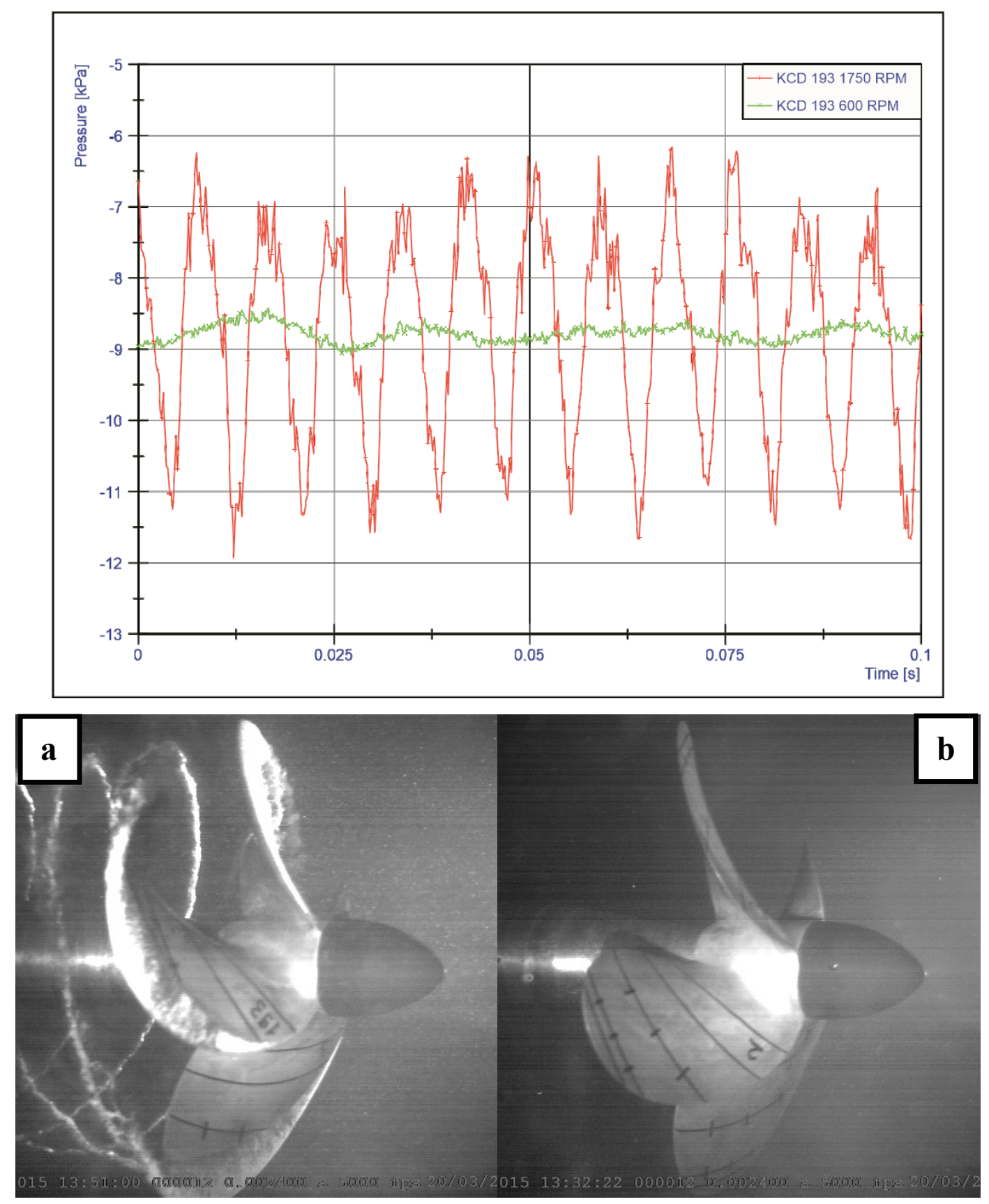

Figure 20 Synchronous time domain pressure signals and cavitation observations under atmospheric pressure for KCD 193 behind ECT1 for 1750 RPM (a) and 600 RPM (b)

Figure 20 is the reproduction of the test data used for Figure 14 using synchronized pressure transducer time signals and cavitation observations. The propeller is cavitating heavily for the condition with propeller speed of 1750 RPM whilst 600 RPM is a non-cavitating condition. The cavitating pressure signal shows significantly higher amplitudes with larger highfrequency irregularities (spikes). Such spikes are attributed to the cavitation collapse as shown in the corresponding cavitation observations by Figure 14 which consequently results in the increase of noise levels over a broad frequency range. 

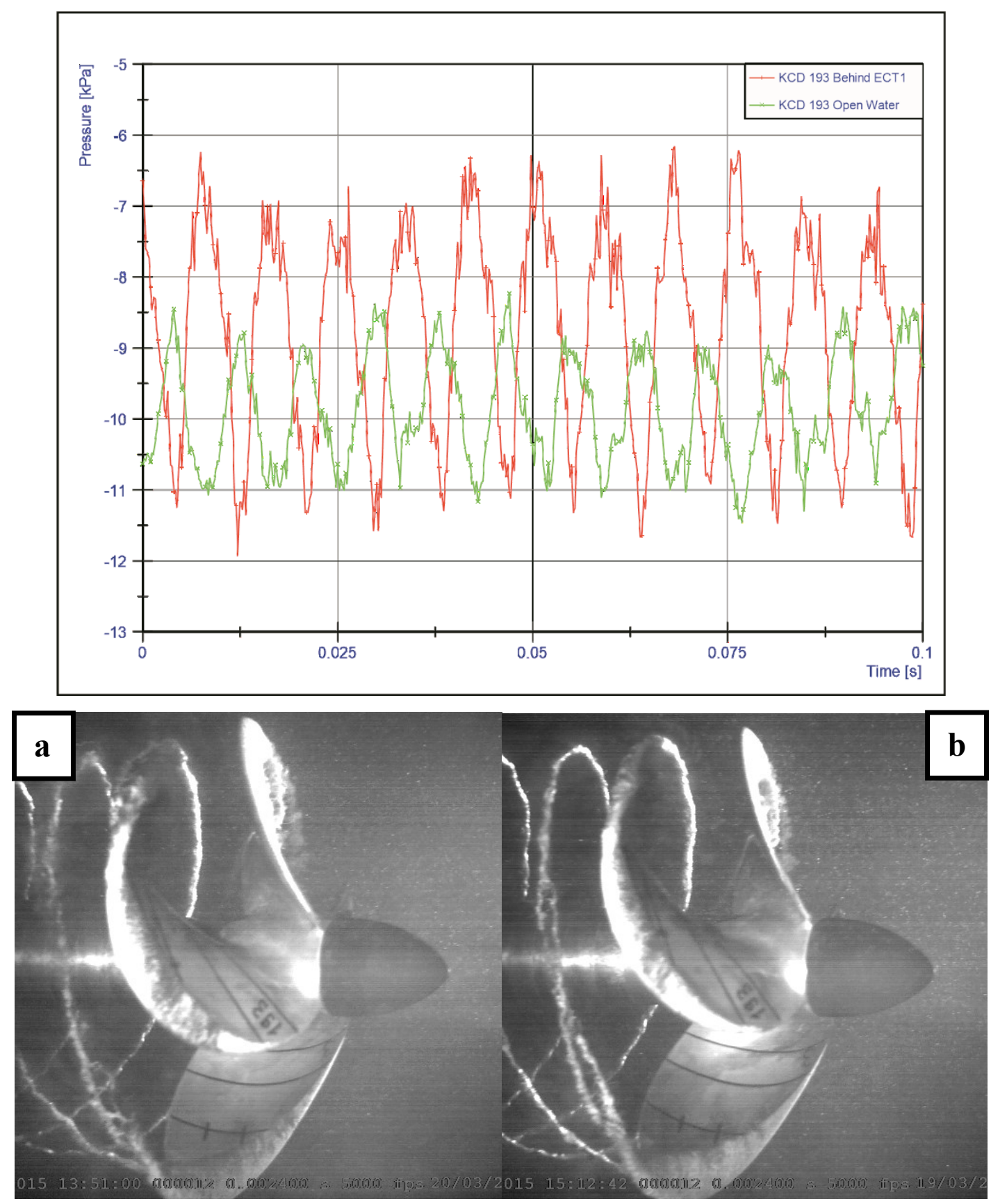

Figure 21 Synchronous time domain pressure signals and cavitation observations under atmospheric pressure for KCD 193 at 1750 RPM behind ECT1 (a) and open water condition (b)

Figure 21 shows differences only due to the wake flow; ECT1 and open water. The pressure time signals differ significantly both in terms of the amplitude and the spikes present in the time signal. The images also support this finding as both tip vortex and sheet cavitation for the propeller in open water condition are more stable, whilst behind ECT1, bursting of a tip vortex cavitation (TVC) due to non-uniform inflow is observed in the slipstream together with unstable sheet cavitation attached to the propeller blade. 

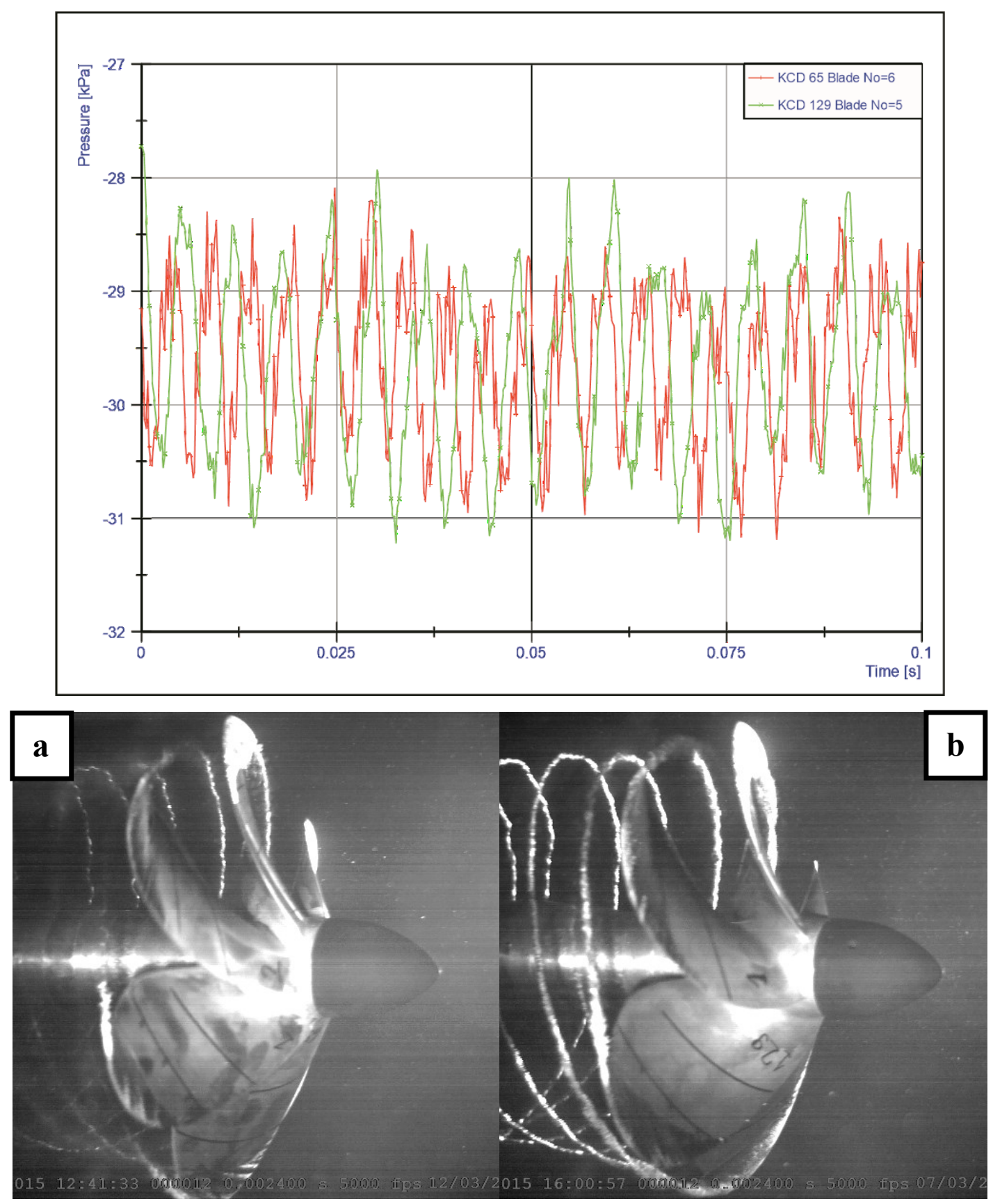

Figure 22 Synchronous time domain pressure signals and cavitation observations under 150 mmHg vacuum behind ECT2 at 2000 RPM for KCD 65 (a) and KCD 129 (b)

The effect of different blade numbers is shown in Figure 22. The thrust load per blade is less for KCD 65, which has the higher number of blades ( 6 blades). KCD 65 is experiencing rather unsteady tip vortex cavitation collapses in the propeller slipstream as shown in the images, which are resulting in spikes in the associated time signals. On the other hand, KCD 129 (5 blades) shows comparatively more stable cavitation and a thicker diameter of tip vortex cavitation. The impact of the cavitation dynamics experienced by both propellers on the 
measured noise levels are presented by Figure 16. KCD 129 emanates higher noise levels at the $1^{\text {st }} \mathrm{BPF}$ and a tip vortex cavitation hump that is shifted towards the lower frequencies with relatively high peak SPL. Whereas, KCD 65 experiences the tip vortex cavitation hump at relatively higher frequencies and with a lower maximum SPL over the hump with more prominent broadband noise dominating the high frequency region. 

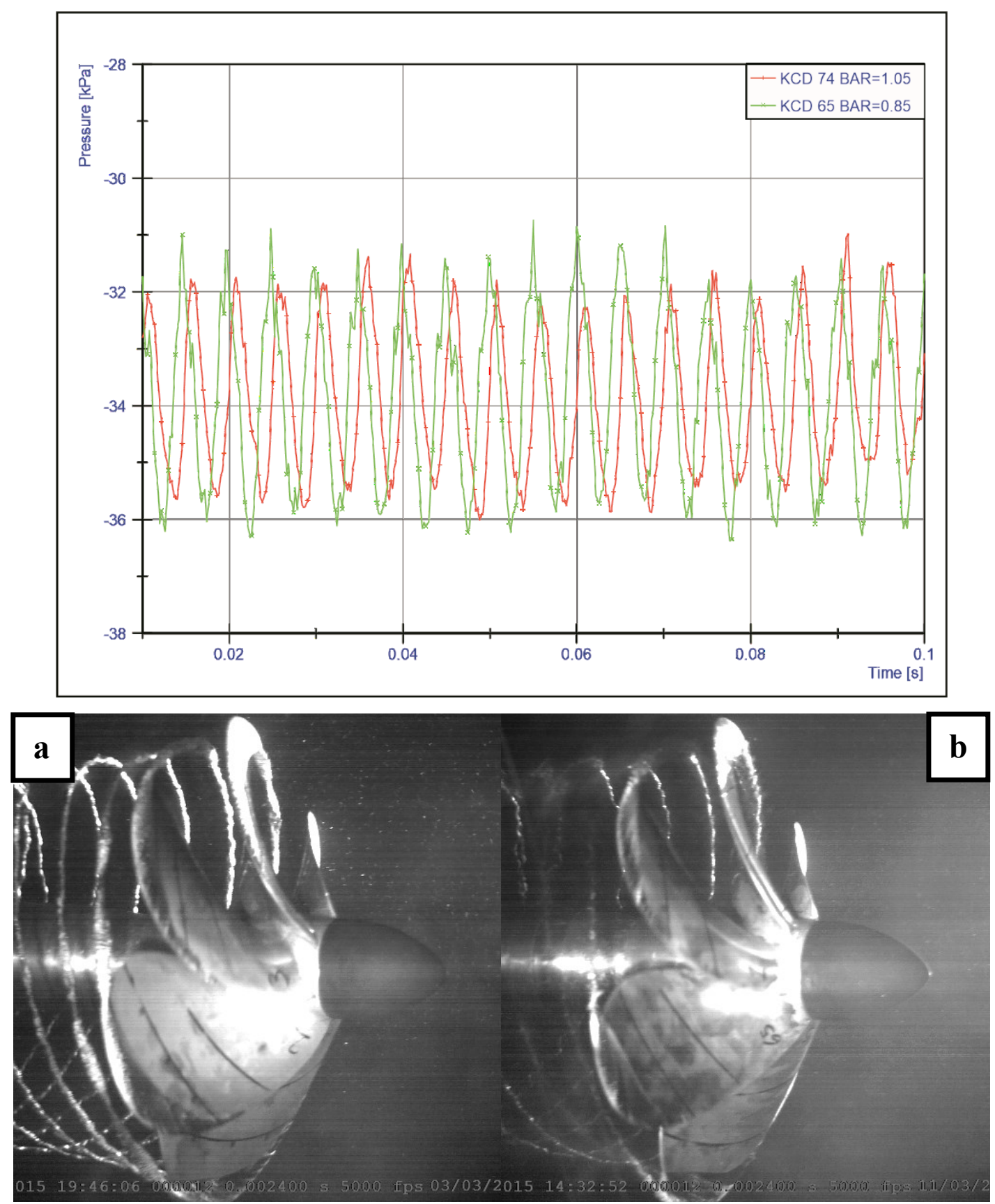

Figure 23 Synchronous time domain pressure signals and cavitation observations under 150 mmHg vacuum behind ECT1 at 2000 RPM for KCD 74 (a) and KCD 65 (b)

Figure 23 presents the effect of the BAR for two 6-bladed propellers. KCD 74 has a higher BAR, hence a lower thrust density. This produces a smaller extent and volume of sheet cavitation and hence a smaller peak to peak pressure signal. The sheet cavitation produced on KCD 65, with its higher thrust loading, is relatively more unstable, showing foamy collapsing behaviour in the slipstream, whilst the tip vortex cavitation of KCD 74 is rather stable but thicker in diameter. The effect of the cavitation dynamics is also evident in the measured SPL as presented by Figure 17. The SPL measured for KCD 74 is relatively higher from $200 \mathrm{~Hz}$ to 
$800 \mathrm{~Hz}$ which can be attributed to the thicker tip vortex cavitation experienced by the propeller. For KCD65, instability of tip vortex cavitation and relatively smaller core diameter results in the spectral hump to shift towards the higher mid-frequencies and at a lower local maximum SPL. Furthermore, the foamy collapse of the tip vortex produced broadband noise and spectral elevation in high frequencies.
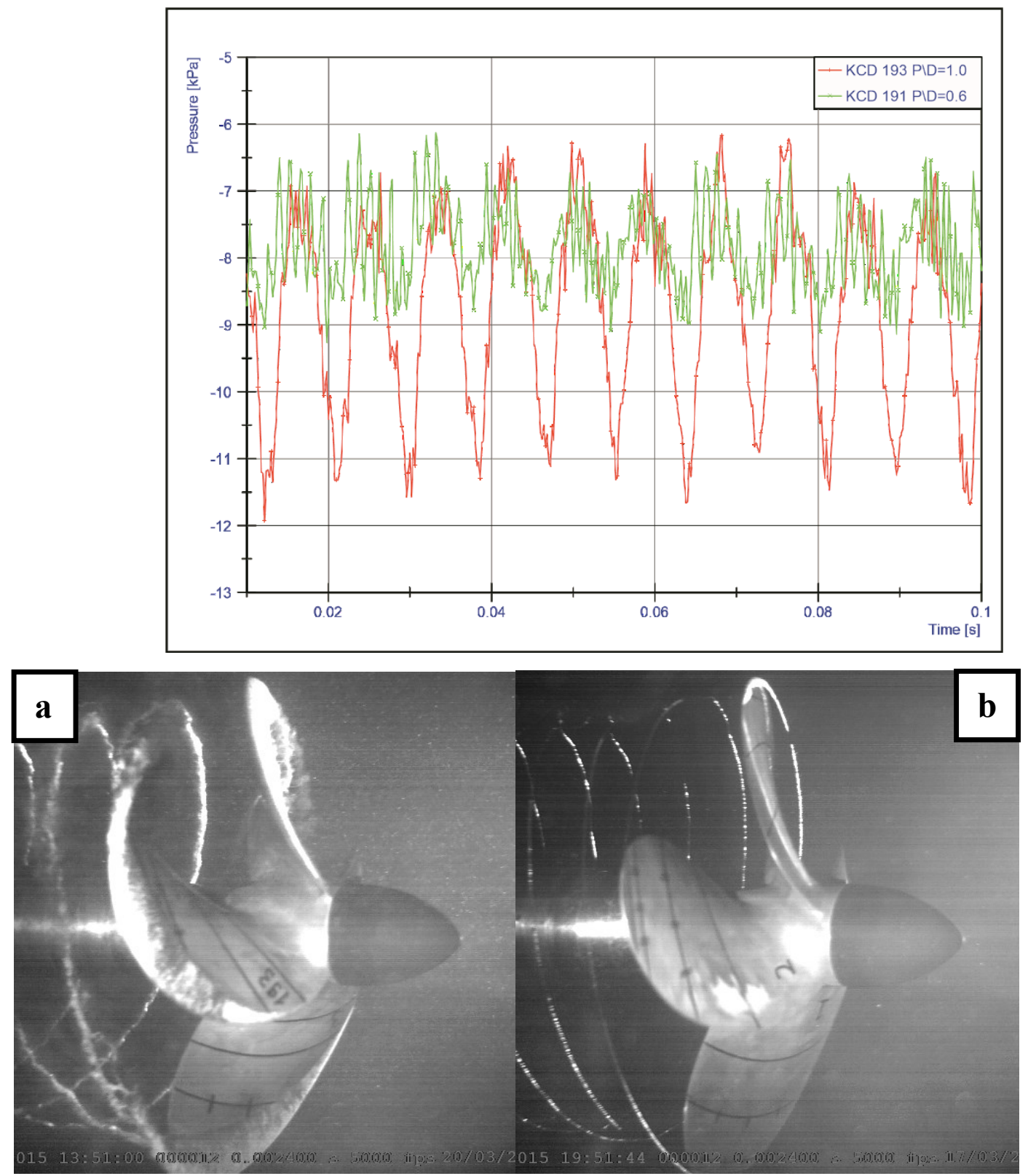

Figure 24 Synchronous time domain pressure signals and cavitation observations under atmospheric pressure behind ECT1 at 1750 RPM for KCD 193 (a) and KCD 191 (b).

Figure 24 shows the pressure time series and cavitation images for two different $\mathrm{P} / \mathrm{D}$ ratios. At the same J-value, KCD 193 is operating at a higher loading than KCD191. Consequently, it generates a larger extent of sheet cavitation as well as unsteady tip vortices travelling into the 
slipstream with foamy appearance. The pressure time signal for the KCD 193 experiences a larger peak to peak amplitude, mainly due to the sheet cavitation volume and irregularities due to the cloudy collapse of the tip vortex cavitation. KCD 191 operates at a much lighter loading and thus produces no sheet cavitation; only intermittent tip vortex cavitation both attached to the propeller and in the slipstream. The tip vortex cavitation is bursting as it detaches from the propeller resulting on a very spiky pressure time signal. The impact of the cavitation dynamics is also evident in the measured SPL as presented by Figure 18 in which the spectra by KCD 191 is significantly lower at frequencies lower than 600 and higher than 2000 due to absence of the sheet cavitation. 


\subsection{Joint time-frequency analysis of fluctuating pressures and URN of propellers}

The acquired pressure time signal data, described in the previous section, has then been fed into the Joint Time-Frequency Analysis (JTFA) tool in order to understand the relationship between time events of the pressure pulse signals and cavitation presence and corresponding frequency domain response. The JTFA tool does this through a spectrogram, which can be used to gain enhanced understanding for the frequency region that certain types of cavitation contribute into.

For this purpose, the JTFA technique is used as a tool to establish a bridge between the time domain and frequency response. The developed methodology is demonstrated through comparisons of major propeller design and operational parameters to enhance the understanding of their roles as noise producing mechanisms of the propeller cavitation. The quadratic joint time-frequency representation for the input signal is calculated using ShortTime Fourier Transform (STFT) spectrogram. For STFT, first pressure pulse measurements that has been acquired at $5000 \mathrm{~Hz}$ for 5 seconds is first imported. The signal is cropped to be left with only the first 0.1 second of the signal as cavitation observations were recorded only for the first 0.1 seconds. For the analysis Hanning type window is used with a length of 64 . The data is analysed using only one time step and 512 frequency bins. 


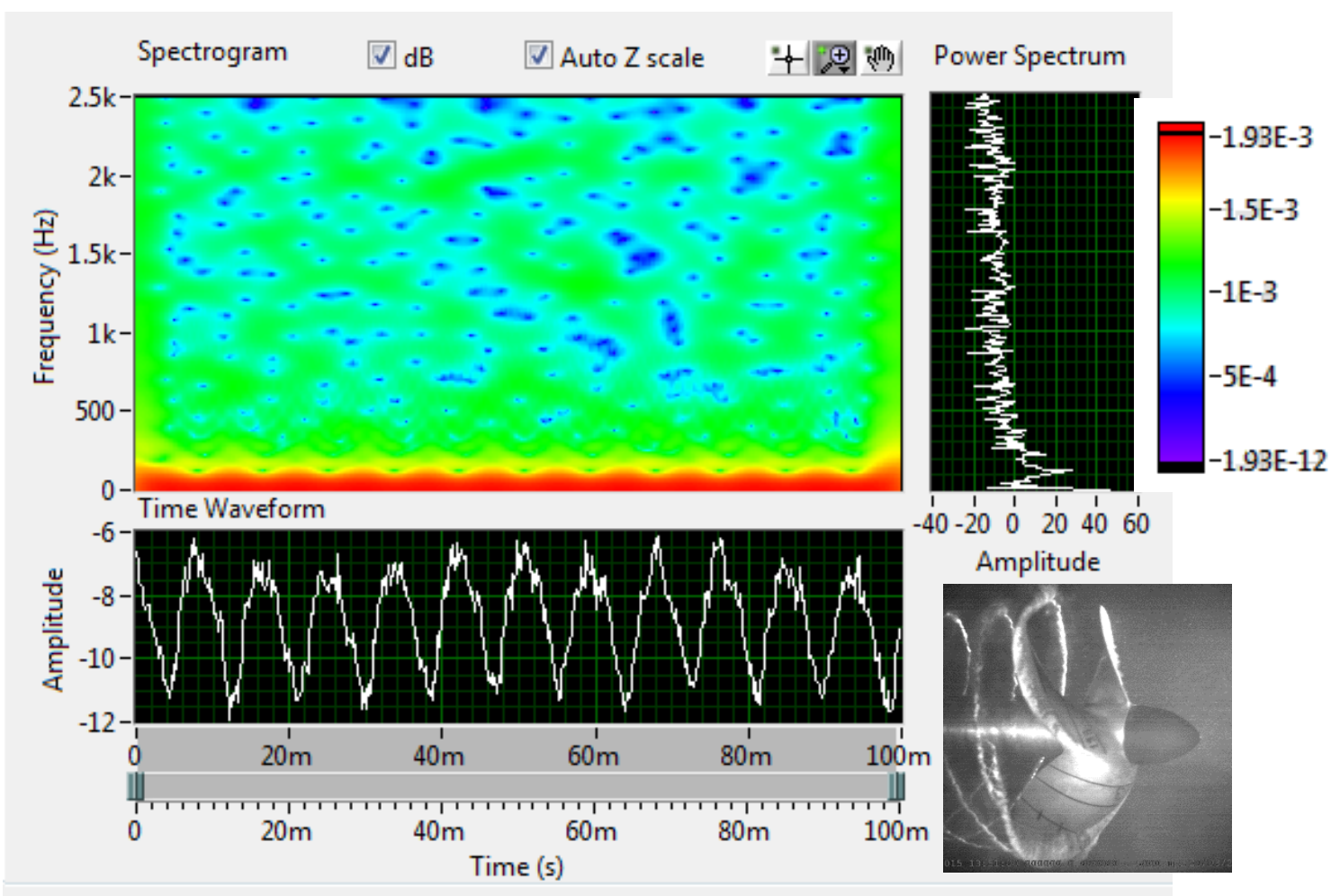

Figure 25 Jointtime-frequency analysis of KCD 193 propeller behind ECT1 under atmospheric condition at 1750 RPM

Figure 25 presents pressure recordings and analysis results of KCD 193 propeller for its most heavily cavitating condition. The spectrogram indicates that the spikes present in the time signal contribute over a broad spectral range. Although the analysis is limited to $2.5 \mathrm{kHz}$ since the pressure transducer signal is sampled at $5 \mathrm{kHz}$, the noise data from the hydrophone analysis, which is shown in Figure 14, also indicates contributions from the cavitation dynamics at higher frequencies. Overall the spectrogram has only very small areas occupied by the blue colour referring to the lower amplitude activity while it is dominated by the greenish colour corresponding to the higher amplitude activities in the FFT at broadband range. 


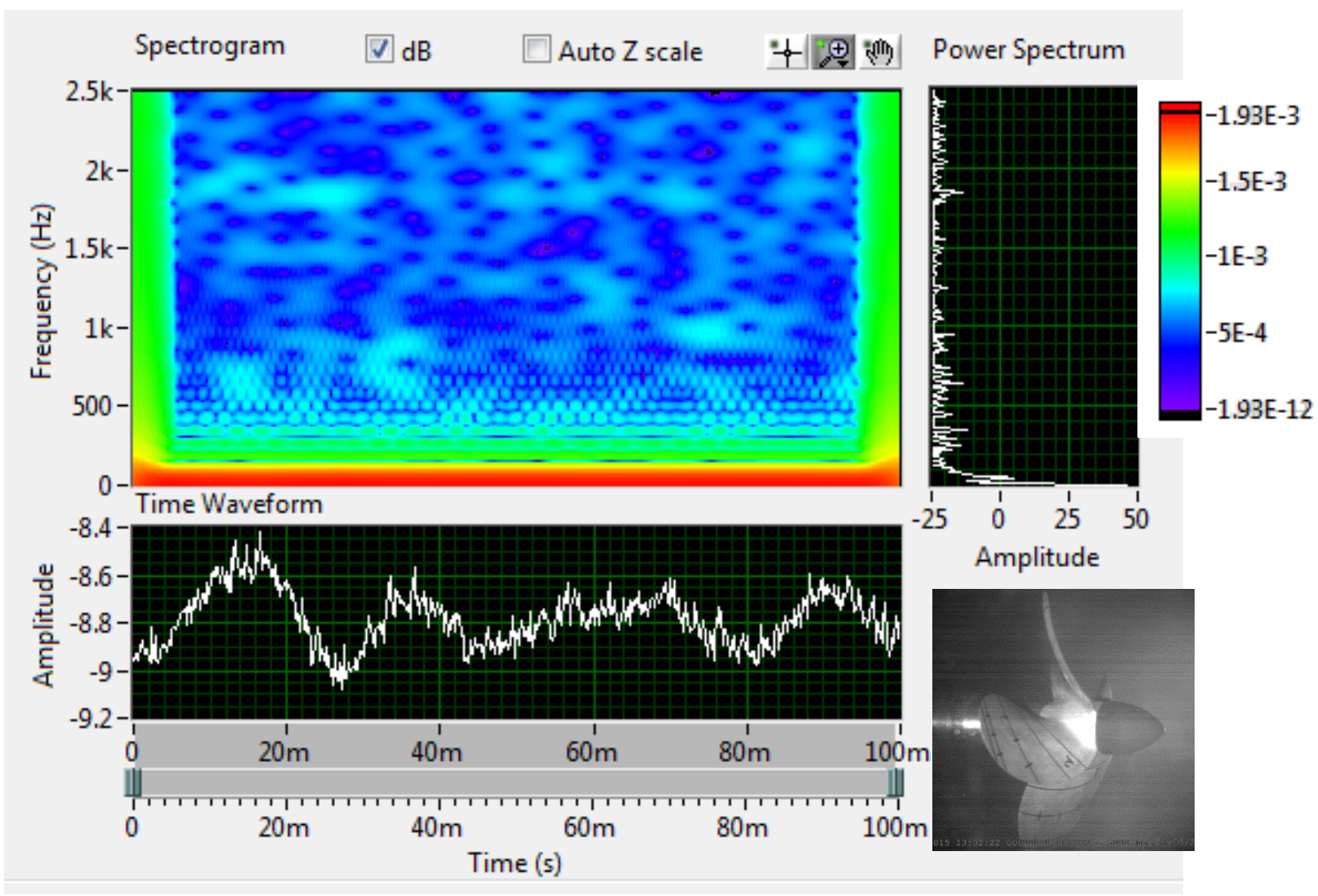

Figure 26 Joint time-frequency analysis of KCD 193 propeller behind ECT1 under atmospheric codition at 600 RPM

Figure 26 shows the JTFA analysis of KCD 193 behind ECT1 wake at 600 RPM corresponding to a non-cavitating condition. As a consequence, and shown in Figure 26, not only the peak to peak amplitudes have been significantly reduced but also the overall amplitude levels in the spectral domain have been considerably reduced, as indicated by the dominant blue colouring (low levels). A distinct peak is shown in the FFT results between $1.5-2 \mathrm{kHz}$ which is also captured in Figure 14 by the spectral levels of the corresponding hydrophone measurements. The peak may be a consequence of singing of the propeller. 


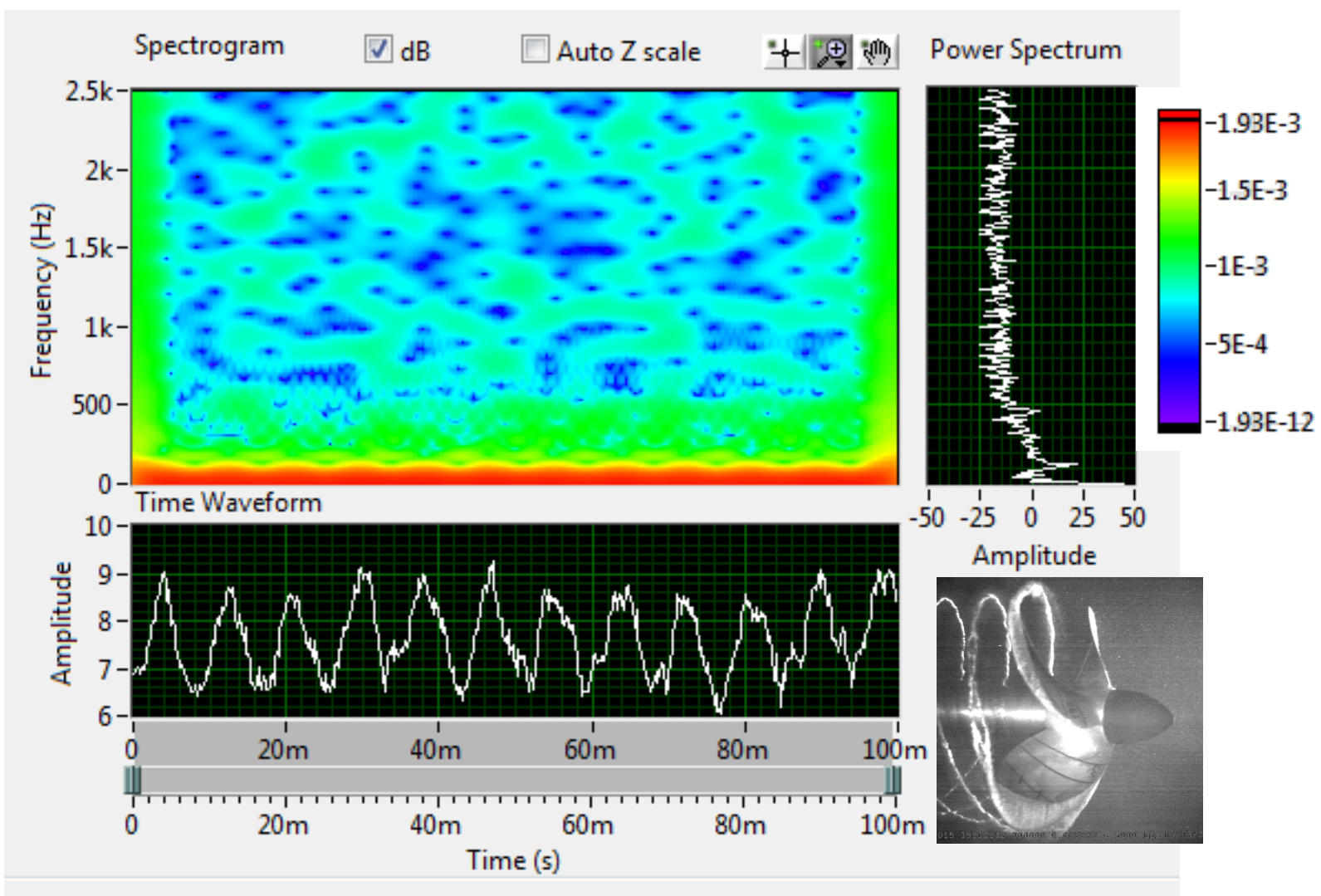

Figure 27 Joint time-frequency analysis of KCD 193 propeller in open water condition under atmospheric codition at 1750 RPM

Figure 27 shows the pressure recording and analysis results of KCD 193 propeller at 1750 RPM but in open water condition. Overall the analysis of the results shows a reduction at the blade rate and broadband spectra in terms of the amplitudes when compared to Figure 25. Moreover, the previous comment is supported by the spectrogram colour map which is occupied by bluer colour footprints over the broadband range. The above findings justify the use of JTFA by further confirming the results presented in Figure 15. One interesting observation, which can be made from the spectrogram is the contour oscillations at the blade rate frequency. The spectrogram presents yellow colour during the peak in the time signal that coincides with the passage of a propeller blade which indicates the presence of a large cavitation volume. Following the passage of the blade, contour colour changes to green in conjunction with the trough of the time signal which is experienced by moving propeller blade. Another important aspect of Figure 27 is the hump in the narrow band frequency range from 160 to $400 \mathrm{~Hz}$ which is again present in the noise measurements as shown in Figure 15. Overall the spectrogram shows a rather denser green colour in Figure 25 indicating the higher emanated sound pressure levels in comparison to the Figure 27. 


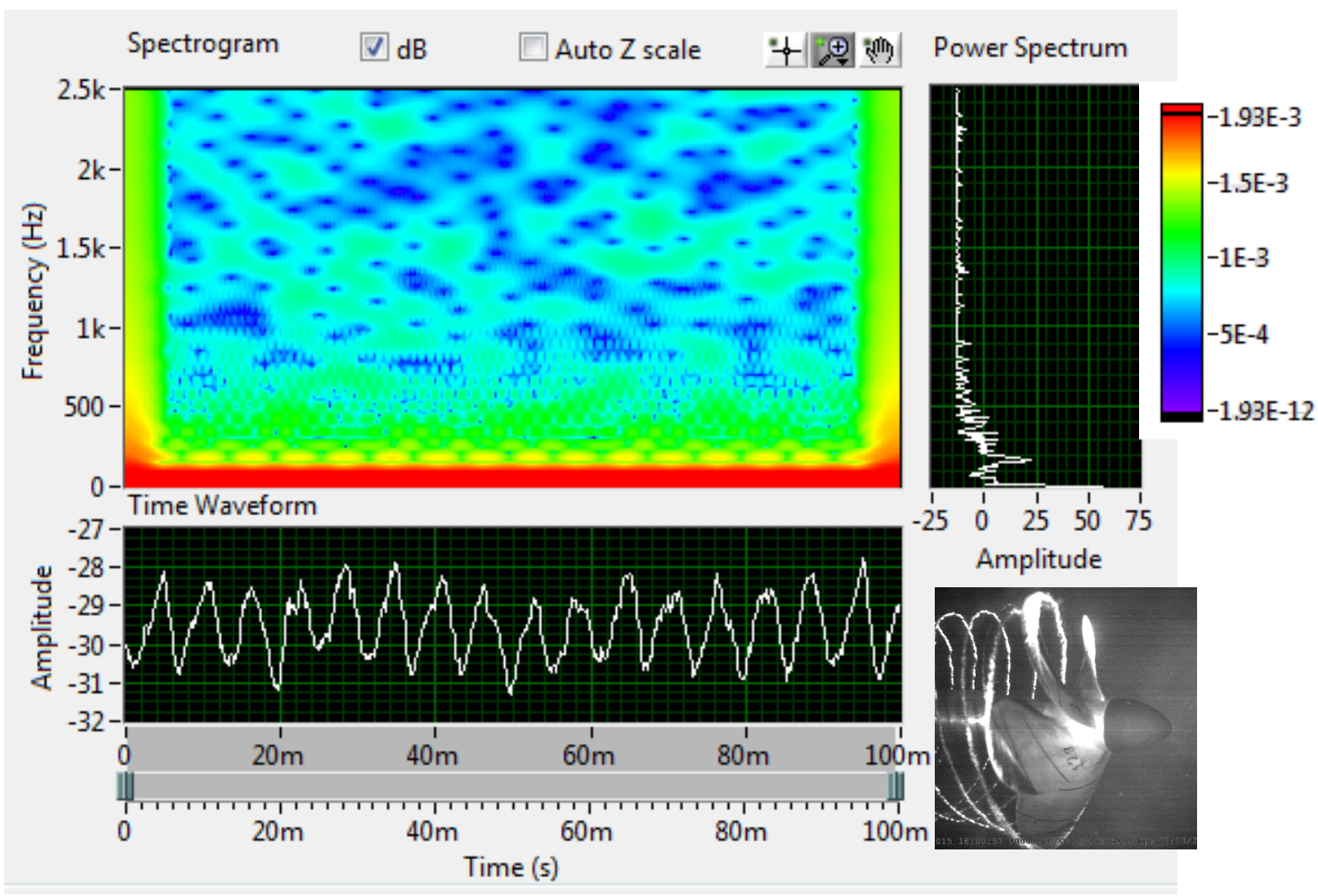

Figure 28 Joint time-frequency analysis of KCD 129 propeller behind ECT2 under $150 \mathrm{mmHg}$ vacuum at 2000 RPM (5 blades)

Figure 28 represents the analysis of one of the test runs to investigate the effect of blade number. The results of the URN measurements with KCD 129 (5 blades) and KCD 65 (6 blades) for this particular test run have already been shown in Figure 16. In this condition, the propeller generates a stable TVC. Both the spectrogram and power spectrum results show elevated pressure levels in the 200 to $600 \mathrm{~Hz}$ frequency range as represented by the green colour. This elevation also reveals itself in the noise measurements by hydrophone as can be seen in Figure 16. Another interesting aspect of Figure 28 is the clear presence of the blade passages in the spectrogram by the green and yellow pulsations present at the blade rate frequency $(167 \mathrm{~Hz})$. 


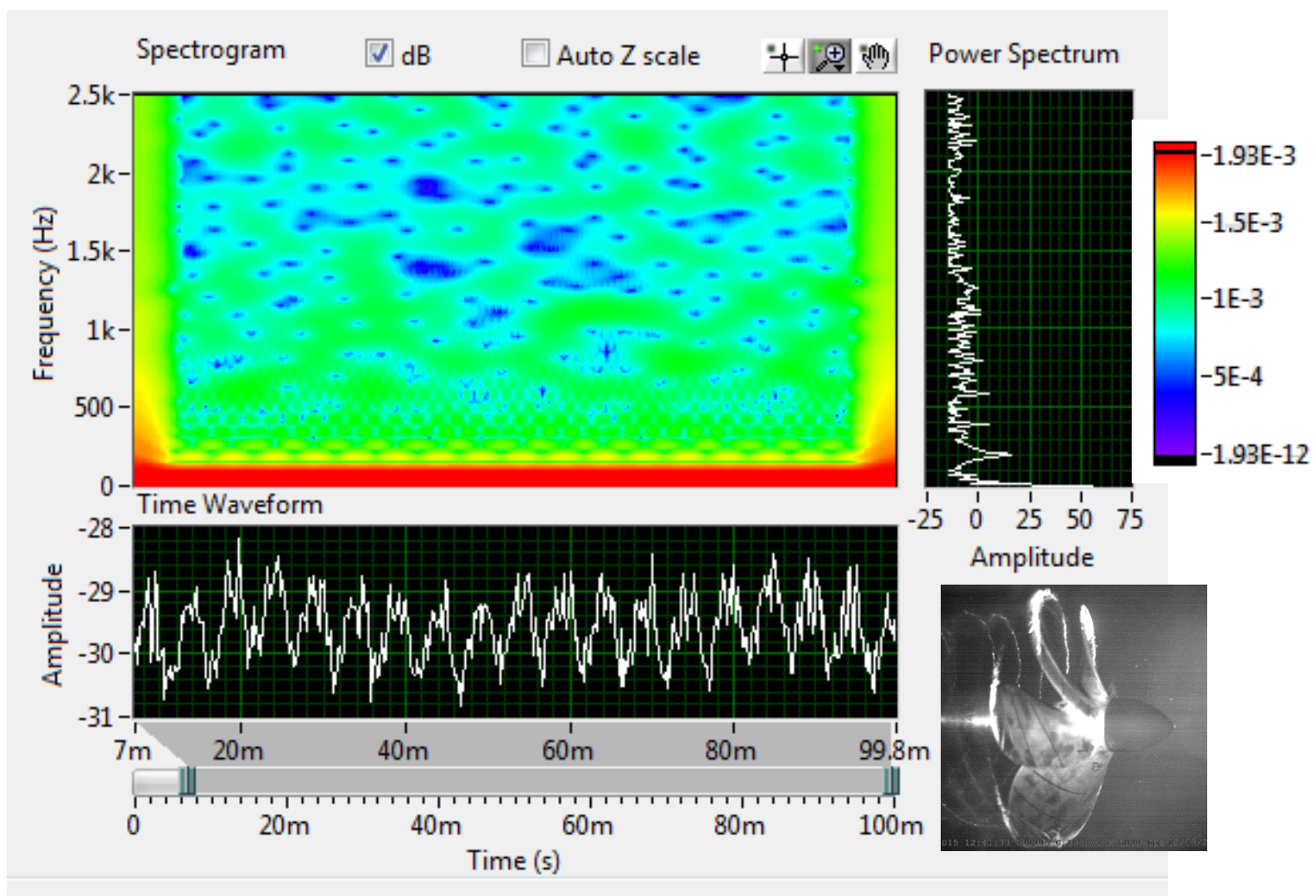

Figure 29 Joint time-frequency analysis of KCD 65 propeller behind ECT1 under $150 \mathrm{mmHg}$ vacuum at 2000 RPM

Figure 29 is the JTFA analysis results of the test run with KCD 65 propeller which is the 6bladed counterpart of KCD 129. The comparative results of the noise measurements with both propellers (i.e. KCD 129 and KCD 65) for the same test run have been already shown in Figure 16 , which can be combined with the JTFA results during the discussion. In this test run KCD 65 experiences a larger extent of sheet cavitation combined with a rather unstable foamy tip vortex cavitation in the slipstream. The larger volume of the sheet cavitation results in more dramatic collapse and rebounding resulting in higher cavitation volume acceleration and hence relatively spikier time signals. The comparison of Figure 28 and Figure 29 does suggest that the SPLs of KCD 129 appear to be higher up to $600 \mathrm{~Hz}$ in comparison to the results of KCD 65. The rest of the frequency range, however, indicates higher noise levels radiated by KCD 65. This is in good agreement with the earlier presented spectral levels in Figure 16. 


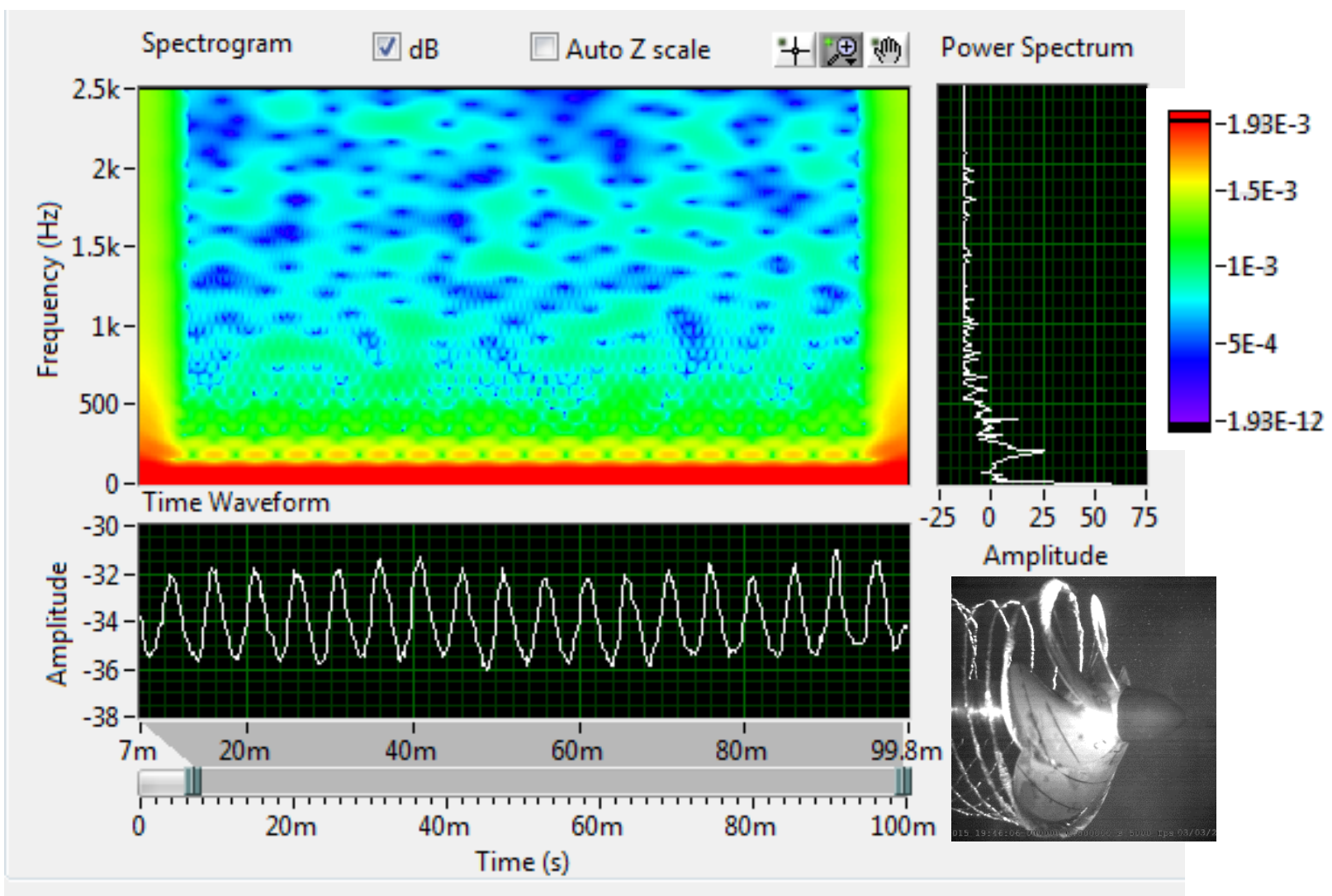

Figure 30 Joint time-frequency analysis of KCD 74 propeller behind ECT1 under $150 \mathrm{mmHg}$ vacuum at 2000 RPM

Figure 30 represents the JTFA results for KCD 74 selected to discuss the effect of the BAR on the URN levels. KCD 74 has a higher BAR compared to its counterpart propeller KCD 65. Hence the interpretation of the results should be combined with the URN measurement results of the same propeller which have been presented in Figure 28 as discussed earlier. KCD 74 at this test condition does have a rather continuous green background of spectrogram results in the 200 to $600 \mathrm{~Hz}$ range but also has a region dominated by the blue in the high frequency. The propeller experiences a stable TVC with dynamic nature as it can be seen to experience some volume fluctuation as it travels through the slipstream. The peak at $200 \mathrm{~Hz}$ coincides with the $\mathrm{BPF}$ and is represented by the green and yellow/orange fluctuations at lower part of the spectrogram. Figure 30 confirms the trend in the URN measurements observed in Figure 17 showing all dominant spectral features of the cavitating propeller. 


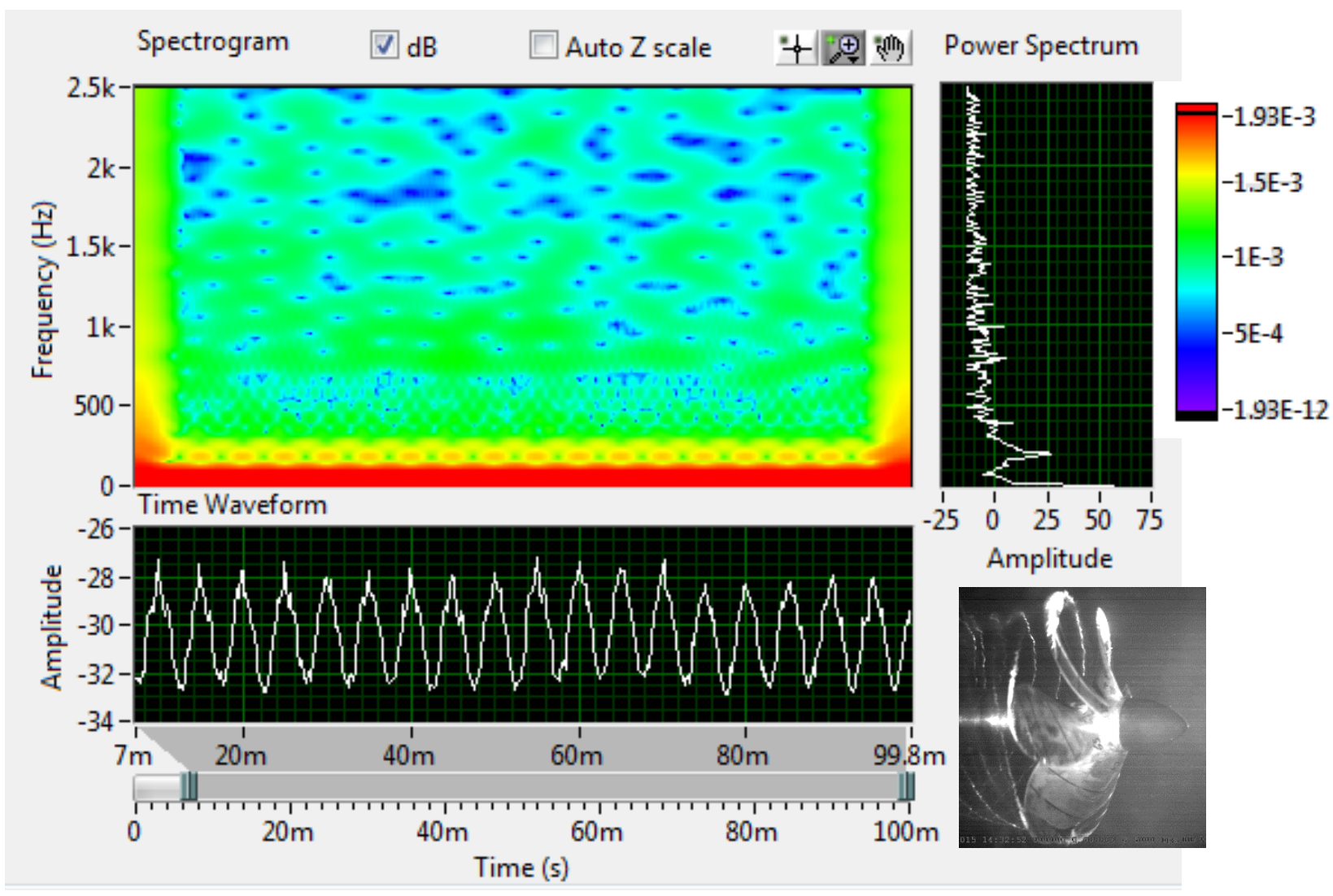

Figure 31 Joint time-frequency analysis of KCD 65 propeller behind ECT1 under $150 \mathrm{mmHg}$ vacuum at 2000 RPM

Figure 31 represents the JTFA results of KCD 65 having the lower BAR. While the background colour of the spectrogram is exactly the same up to the $1^{\text {st }} \mathrm{BPF}(200 \mathrm{~Hz})$ for both KCD 65 and KCD 74 propellers, the spectrogram shows relatively larger extent of blue colour in the 200 to $600 \mathrm{~Hz}$ region for the $\mathrm{KCD} 65$. However, thereafter the colour code shows rather greener background, which reveals itself in the power spectrum as the broadband spiky components. In light of the above comments and considering the trend in the URN spectrum as discussed in Figure 17, the results of the JTFA confirms the analysis results of the URN. This is further supported by the cavitation observations presented in Figure 23. 


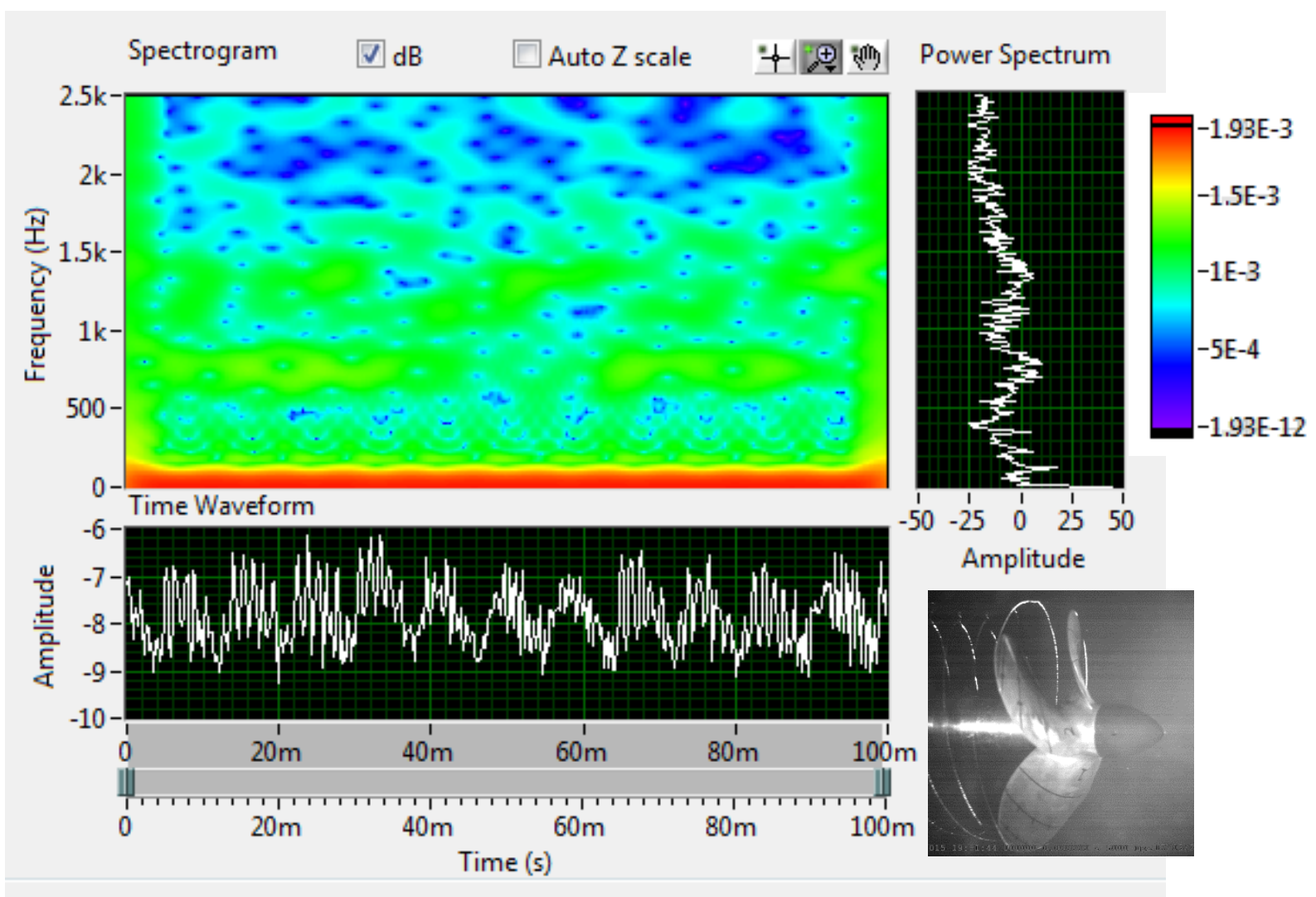

Figure 32 Joint time-frequency analysis of KCD 191 propeller behind ECT1 under atmospheric condition at 1750 RPM

Figure 32 represents the JTFA results of KCD 191 propeller behind wake ECT1 at 1750 RPM. The propeller experiences a thin TVC cavitation that collapses in the slipstream as discussed in Figure 24. The spectrogram shows a rather blue region for the frequencies higher than 1.5 $\mathrm{kHz}$ while the rest of the frequency range between 200 to $1.5 \mathrm{kHz}$ has rather green background, which is also present in the power spectrum analysis part of the JTFA by the elevated levels. The impact of the TVC is displayed as large spikes in the pressure time signal at the exact time of the experienced collapse. These time domain spikes are reflected as continuous green coloured zones on the spectrogram at two distinct frequency ranges: one being at 400 to 1000 $\mathrm{Hz}$ and other at 1000 to $2000 \mathrm{~Hz}$ range. The reflection of these zones is also clearly visible by two distinct humps in the SPL spectrum of KCD 191 propeller as shown in Figure 18. The originating cause of these humps thus can be attributed to the TVC and its dynamics with the aid of quantitative proof of the cavitation observations in conjunction with the JTFA approach implemented. As experienced in this example such a case, where there is only one type of cavitation with intermittent nature, presents a perfect example for the merits of the implemented advanced analysis approach. 


\section{Conclusion}

Based on the systematic propeller cavitation noise data produced in this study, the capability of the implemented analysis methodology is demonstrated for advanced cavitation-induced noise analysis. The main objective of the proposed methodology is to enhance the understanding of the cavitation-induced noise phenomenon. This has been achieved by making use of the synchronised fluctuating pressure signals and cavitation observations in time-domain which is combined with the JTFA technique. The implemented procedure has demonstrated the impact of the important propeller design and operational parameters with regards to the URN phenomenon. Furthermore, the careful investigation of the recorded data revealed the importance of the observed instantaneous cavitation dynamics on the time pressure signals and their consequent impact in the frequency domain.

The frequency domain results of the hydrophone measurements clearly indicated that cavitation is the dominating noise source following its inception. The wake non-uniformity is a vital parameter to consider for the standard series propeller noise tests since the cavitation dynamics experienced due to the flow irregularity is one of the main sources of the emanated noise levels. Moreover, the cavitation type experienced by any propeller is highly influential on the emanated noise levels as well as the frequency region for which a certain type of cavitation contributes into.

The synchronization of the pressure pulse measurements and cavitation observations enabled interpretation of the influence of cavitation events on the pressure time signals. The repetitive major cavitation events and relatively instantaneous dynamic cavitation collapse occurrence has been observed to be the main driving mechanism causing fluctuations and spikes within the measured pressure signals.

Finally, in order to be able to understand the effect of those spikes and fluctuation in the time domain, a Joint Time-Frequency Analysis tool is utilized to establish a bridge between the time series results and their frequency responses. The JTFA provides the colour contoured spectrogram, which is then converted to the frequency response. The frequency response, in conjunction with the synchronized cavitation observations enables the interpretation of frequency region that certain cavitation phenomenon contributes into.

Overall, it can be deduced from the presented analysed data that the tip vortex cavitation results in elevation of the SPLs in mid-frequency regions and reveals itself in hump shaped noise 
signature. The exact frequency region that tip vortex cavitation contributes into changes with its size and dynamics experienced by it. The tip vortex cavitation with larger core diameter results in the hump to shift to relatively lower frequencies with higher maximum SPL. Sheet cavitation mainly elevates the SPL' at the blade rates and in the high frequencies. The contribution of the sheet cavitation at the blade rates is due to its volume and sheet cavitation volume variation over time. The high-frequency elevation is mainly due to sheet cavitation collapse and rebounding phenomenon. Moreover, the

\section{Acknowledgments}

The research constituting the basis for this study has been supported by the European Union $7^{\text {th }}$ Framework Programme (FP7) under grant agreement No: 314394 through the SONIC project.

\section{Appendix A. Synchronized fluctuating pressures and cavitation observation videos}

The video captures of the synchronized fluctuating pressure pulses and cavitation observation videos are provided within this appendix.

Click here to download Video: KCD193_W1_Atm_1750RPM_600RPM.mov

Video 1.: Synchronous time domain pressure signals and cavitation observation video under atmospheric pressure for KCD 193 behind ECT1 for 1750 RPM (left) and 600 RPM (right)

Click here to download Video: KCD193_W1_OW_Atm.mov

Video 2.: Synchronous time domain pressure signals and cavitation observations under atmospheric pressure for KCD 193 at 1750 RPM behind ECT1 (left) and open water condition (right)

Click here to download Video: KCD65_KCD129_W2_150mmhg.mov

Video 3.: Synchronous time domain pressure signals and cavitation observations under $150 \mathrm{mmHg}$ vacuum behind ECT2 at 2000 RPM for KCD 65 (left) and KCD 129 (right)

Click here to download Video: KCD65_KCD74_W1_150mmhg_1750.mov 
Video 4.: Synchronous time domain pressure signals and cavitation observations under $150 \mathrm{mmHg}$ vacuum behind ECT1 at 2000 RPM for KCD 74 (left) and KCD 65 (right)

Click here to download Video: KCD193_KCD191_W1_atm.mov

Video 5.: Synchronous time domain pressure signals and cavitation observations under atmospheric pressure behind ECT1 at 17500 RPM for KCD 193 (Left) and KCD 191 (Right)

\section{Reference}

Aktas, B., 2017. A Systematic Experimental Approach to Cavitation Noise Prediction of Marine Propellers. Newcastle University, PhD Thesis.

Angelopoulos, A., Fitzsimmons, P.A., Odabasi, A.Y., 1988. A Semi-Empirical Method for Propeller Broad-Band Noise. Report No: W1906, British Maritime Technology Limited, p. 14.

Atlar, M., 2011. Recent upgrading of marine testing facilities at Newcastle University, in: The 2nd International Conference on Advanced Model Measurement Technology for the EU Maritime Industry. Newcastle upon Tyne, UK, 4-6 April, pp. 1-32.

Bosschers, J., 2009. Investigation of Hull Pressure Fluctuations Generated by Cavitating Vortices. Proc. First Symp. Mar. Propulsors.

Breslin, J.P., Andersen, P., 1996. Hydrodynamics of Ship Propellers, Cambridge Ocean Technology Series. Cambridge University Press. doi:10.1017/CBO9780511624254

Bretschneider, H., Bosschers, J., Choi, G.H., Ciappi, E., Farabee, T., Kawakita, C., Tang, D., 2014. Specialist Committee on Hydrodynamic Noise, Final Report and Recommendations to the 27th ITTC. Copenhagen, Sweden.

Burrill, L.C., Emerson, A., 1962. Propeller Cavitation-Further Tests on 16in Propeller Models in the King's College Cavitation Tunnel. Trans. North East Coast Inst. Eng. 79:, 295-320.

Carlton, J., 2012. Marine Propellers and Propulsion. Butterworth-Heinemann.

Emerson, A., Sinclair, L., 1978. Propeller Design and Model Experiments. Trans. North East 
Coast Inst. Eng. Shipbuild. 94:, 199-234.

Frisk, G. V., 2012. Noiseonomics: The relationship between ambient noise levels in the sea and global economic trends. Sci. Rep. 2, 2-5. doi:10.1038/srep00437

Gawn, R.W.L., Burrill, L.C., 1957. Effect of Cavitation on the Performance of a Series of 16 in. Model Propellers. Inst. Nav. Archit. -- Trans. 99:, 690-718.

Holden, K., 1981. Effect of propeller design parameters on noise induced by cavitation, in: Nilsson, A.C., Tyvand, N.P. (Eds.), Noise Sources in Ships I:Propellers. Nordforsk, Miljovardsserien, Sweden.

ITTC, 2011. Model - Scale Cavitation Test, in: Recommended Procedures and Guidelines, 7.502-03-03.1. 26th ITTC Specialist Committee on Scaling of Wake Fields, p. 9.

ITTC, 1987. Cavitation committee report, 18th International Towing Tank Conference. Kobe, Japan, 18th International Towing Tank Conference, October.

Konno, A., Wakabayashi, K., Yamaguchi, H., Maeda, M., Ishii, N., Soejima, S., Kimura, K., Yamguchi, H., Maeda, M., Ishii, N., Soejima, S., Kimura, K., 2002. On the Mechanism of the Bursting Phenomena of Propeller Tip Vortex Cavitation. J. Mar. Sci. Technol. 6, 181-192. doi:10.1007/s007730200006

Korkut, E., 1999. An Investigation Into The Scale Effects on Cavitation Inception and Noise in Marine Propellers. School of Marine Science and Technology Technology, Newcastle University, Philosophy of Doctorate Thesis.

Kuiper, G., 2010. New developments and propeller design. J. Hydrodyn. Ser. B 22, 7-16. doi:10.1016/S1001-6058(09)60161-X

McKenna, M.F., Wiggins, S.M., Hildebrand, J.A., 2013. Relationship between container ship underwater noise levels and ship design, operational and oceanographic conditions. Sci. Rep. 3, 10. doi:10.1038/srep01760

MCR, 2011. Cruise report for research projects conducted from R/V Song of the Whale in French and UK waters of the English Channel, in: Marine Conservation Research International, Research Conducted from R/V Song of the Whale in French and UK Waters of the English Channel, May - June 2011. Essex, UK, p. 18. 
National Instruments, 2011. DIAdem, Data Mining, Analysis, and Report Generation. User Man. 373082J-01, 122.

National Instruments, 2003. National Instruments, LabView.

Odabasi, A., Fitzsimmons, P., 1978. Alternative methods for wake quality assessment. Int. Shipbuild. Prog. 25, February 1978.

Oossanen, P. van, 1971. A Method for Minimizing the Occurence of Cavitation on Proplellers in a Wake. Int. Sh. Build. Prog. 18, 321-333.

Pennings, P., Westerweel, J., Terwisga, T. van, 2016. Cavitation tunnel analysis of radiated sound from the resonance of a propeller tip vortex cavity. Int. J. Multiph. Flow 83, 1-11. doi:10.1016/J.IJMULTIPHASEFLOW.2016.03.004

Pereira, F., Felice, F. Di, Soave, M., Salvatore, F., 2004. Experimental Investigation of a Cavitating Propeller in Non-Uniform Inflow. 25th ONR Symp. Nav. Hydrodyn.

Richardson, W.J.W., Greene, C.R., Jr., Malme, C.I.C., Thomson, D.H.D., Jr, C.G., Malme, C.I.C., Thomson, D.H.D., 2013. Marine mammals and noise. Elsevier Science.

Ross, D., 1976. Mechanics of Underwater Noise. Peninsula Publishing, California, USA. doi:10.1016/B978-0-08-021182-4.50014-3

Sharma, S.D., Mani, K., Arakeri, V.H., 1990. Cavitation noise studies on marine propellers. J. Sound Vib. 138, 255-283. doi:10.1016/0022-460X(90)90542-8

Stopford, M., 1997. Maritime Economics, Second Edi. ed. doi:10.1016/S0966-6923(98)000210

Tani, G., Viviani, M., Hallander, J., Johansson, T., Rizzuto, E., 2016. Propeller underwater radiated noise: A comparison between model scale measurements in two different facilities and full scale measurements. Appl. Ocean Res. 56:, 48-66. doi:10.1016/j.apor.2016.01.007

Wales, S.C., Heitmeyer, R.M., 2002. An ensemble source spectra model for merchant shipradiated noise. J. Acoust. Soc. Am. 111, 1211-1231. doi:10.1121/1.1427355

White, P., Pace, F., 2010. The Impact of Underwater Ship Noise on Marine Mammals. 1st 
IMarEST Sh. Noise Vib. Conf.

Young, Y.L., Kinnas, S.A., 2001. A BEM for the Prediction of Unsteady Midchord Face and/or Back Propeller Cavitation. J. Fluids Eng. 123, 311. doi:10.1115/1.1363611 